\title{
Community Structure in the United States House of Representatives
}

\author{
Mason A. Porter \\ Department of Physics and Center for the Physics of Information, \\ California Institute of Technology, Pasadena, CA 91125, USA \\ Peter J. Mucha \\ Department of Mathematics and Institute for Advanced Materials, \\ University of North Carolina, Chapel Hill, NC 27599-3250 \\ M. E. J. Newman \\ Department of Physics and Center for the Study of Complex Systems, \\ University of Michigan, Ann Arbor, MI 48109-1120 \\ A. J. Friend \\ School of Mathematics, Georgia Institute of Technology, Atlanta, GA 30332-0160

\begin{abstract}
We investigate the networks of committee and subcommittee assignments in the United States House of Representatives from the 101st-108th Congresses, with the committees connected by "interlocks" or common membership. We examine the community structure in these networks using several methods, revealing strong links between certain committees as well as an intrinsic hierarchical structure in the House as a whole. We identify structural changes, including additional hierarchical levels and higher modularity, resulting from the 1994 election, in which the Republican party earned majority status in the House for the first time in more than forty years. We also combine our network approach with analysis of roll call votes using singular value decomposition to uncover correlations between the political and organizational structure of House committees.
\end{abstract}


PACS: 89.75.Fb, 89.65.-s, 89.75.-k, 07.05.Kf

\section{INTRODUCTION}

Much of the detailed work in making United States law is performed by Congressional committees and subcommittees. This contrasts with parliamentary democracies such as Great Britain and Canada, in which a larger part of the legislative process is directly in the hands of political parties or is conducted in sessions of the entire parliament. While the legislation drafted by committees in the U.S. Congress is subject ultimately to roll call votes by the full Senate and House of Representatives, the important role played by committees and subcommittees makes the study of their formation and composition vital to understanding the work of the American legislature.

The presence of committees in the House endows it with obvious hierarchical levels: individual Representatives, subcommittees, standing committees, and the entire House floor. However, it is desirable to examine social networks in the House of Representatives quantitatively to determine whether it has any additional structure that might relate to collaborative efforts among Representatives, such as correlations or close associations between the members of different committees. The importance of such studies is not merely academic. An understanding of the House as a collaboration network may shed considerable light on the law-making process, as bills often spend time in several different committees and subcommittees while being drafted in preparation for votes on the House floor. For instance, the House's consideration of the No Child Left Behind Act of 2001 involved the Committee on Education and the Workforce, the Subcommittee on Education Reform, the Subcommittee on 21st Century Competitiveness, the Committee on the Judiciary, and the Committee on Rules (setting the terms for the scrutiny of the bill) before being approved by the full House [56]. After the Senate further amended the bill, a conference agreement eventually passed both houses of Congress and the final bill was signed by the President to become public law No. 107-110.

Analyzing the structure of the committee system in the House of Representatives and studying its correlation with the partisanship of its constituent Representatives helps achieve a better understanding of political party competition in Congress. Several contrasting theories of committee assignment have been developed in the political science literature 
(a)

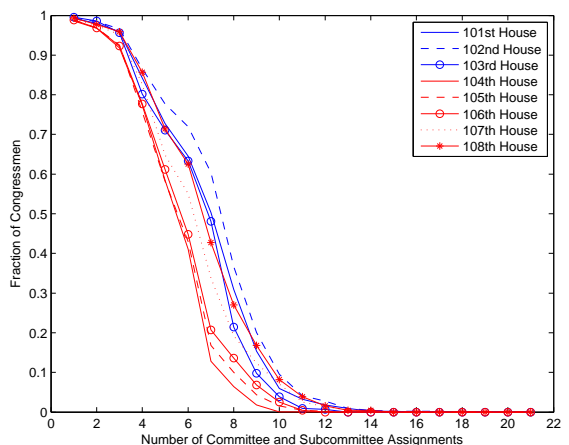

(b)

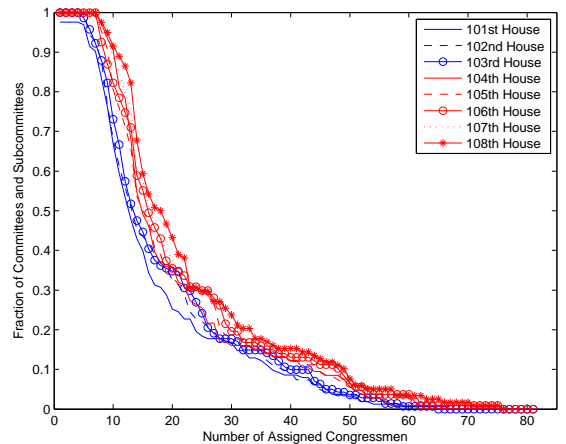

FIG. 1: (Color) Cumulative degree distributions of the 101st-108th U.S. House of Representatives networks defined by committee and subcommittee assignments. (a) Fraction of Representatives versus number of (sub)committee assignments. (b) Fraction of committees and subcommittees versus number of assigned Representatives. In the 104th-108th Houses, all with Republican majorities, the cumulative degree distribution in (a) shifts farther up in each House and that in (b) shows a similar but less pronounced shift. There is no noticeable trend in the Democrat-majority 101st-103rd Houses in (a), but it seems to shift up a bit in (b) to reveal a drift covering all eight Congressional terms we studied.

(mostly through qualitative studies, although there have been some quantitative ones; see [4, 9, 16, 29, 41, 52]), but there is no consensus explanation of how committee assignments are initially determined or how they are modified from one two-year term of Congress to the next. A question of particular interest is whether political parties assign committee memberships essentially at random or if, for instance, one can show using objective analyses that influential Congressional committees are "stacked" with partisan party members.

Our study of the organizational structure of Congress draws on network theory, which provides powerful tools for representing and analyzing complex systems of connected agents. While the quantitative study of real-world networks has a long history in the social sciences (see, for example, the discussions in [33, 60]), such investigations experienced a major expansion in popularity in the late 1990s, in part because of interest in the Internet and online networks. This increased attention has been especially evident in studies of large social, biological, and technological networks, which have relied on major advancements in computer hardware and algorithms to generate novel results [1, 13, 33, 55, 60]. Among the myriad topics that have been studied are evolving social groups [28], collaborations [19], community 
detection [11], and hierarchical organization [49, 51]. It is the modular and hierarchical community structure of networks that primarily concern us in our present study of Congressional committee assignments.

The Congressional networks studied here are examples of collaboration networks, on which there is a considerable body of previous literature. Networks constructed from collaborations between corporate boards of directors [30, 31, 32, 50, 59] are especially germane to the present work, as such collaborations occupy a position in the business world somewhat analogous to that of collaborations between Congressional committee members. Previous studies have shown that board memberships play a major role in the spread of attitudes, ideas, and practices through the corporate world, affecting investment strategies [21], political donations [59], and even the stock market on which a company is listed [48]. Studies of the structure of corporate networks have shed light on the mechanisms and pathways of information diffusion [6, 7, 12], and we believe that the structure of congressional committees may turn out to be similarly revealing.

As we show here, network methods are particularly effective at uncovering structure among committee and subcommittee assignments in Congress, without the need to incorporate any specific knowledge about committee members or their political positions. In a recent article [47], the present authors formulated and briefly examined a number of committee assignment networks, looking, for instance, at the partisanship of the Select Committee on Homeland Security in the 107th House and its connections to other committees. An alternative network perspective on the structure of Congress has been offered by J. H. Fowler [14, 15], who examined the network defined by the cosponsorship of legislation by Members of Congress. In the present work, we compare our previous observations to those for the 108th House and explore the structural changes in the networks that resulted from the 1994 Congressional elections in which the Republican party gained majority control of the House. A detailed technical discussion of the methods used to obtain our results is included in the appendices.

This paper is organized as follows. First, we define the bipartite collaboration networks determined by the assignments of Representatives to House committees and subcommittees. We then investigate the hierarchical and modular structure of these networks using several different community detection methods. We also incorporate singular value decomposition (SVD) analysis of House roll call votes into our study of the House's community structure. 
We provide additional details in two appendices: in Appendix A, we explain the methods used in our SVD analysis of voting patterns; in Appendix B, we give a detailed comparison of several methods for community detection in networks, including our generalization of a recently proposed local detection algorithm [3] to weighted networks.

\section{COMMITTEE ASSIGNMENT NETWORKS IN THE HOUSE}

We represent each of the 101st-108th terms of the U.S. House of Representatives as a separate bipartite (two-mode) network based on assignments of Representatives to committees and subcommittees (henceforth called just "committees" for simplicity). The two types of nodes in these networks correspond to Representatives and committees, with edges connecting each Representative to the committees on which he or she sits. The period we study (1989-2004) spans the political changes following the terrorist attacks of September 11, 2001 [56], as well as the 1994 elections in which the Republican party won majority control of the House for the first time in more than forty years. We construct one network for each two-year Congressional term from data published by the Clerk's Office of the House of Representatives [57], ignoring changes in committee assignments that occur while a term is underway.

Each network includes roughly 440 Representatives (including non-voting Delegates and midterm replacements), about 20 standing committees, and more than 100 subcommittees, with an average of about 6 committee assignments per Representative. Because of the relatively high edge density (about $5 \%$ of possible connections are present), some frequently studied network statistics, such as geodesic path lengths, turn out to be unrevealing in this case. Therefore, we instead focus our attention on the community structure of the networks and associated measures such as modularity and Horton-Strahler numbers. We discuss these analyses in Section III.

With data for eight consecutive Congresses, it is natural to ask how the committee assignment network changes in time. One question of interest is whether the networks contain signs of the so-called "Republican Revolution" of 1994 that ended forty years of Democratic majorities in the House of Representatives, the longest span of single-party rule in Congressional history [27]. That is, can one observe structural differences in the committee assignment networks between the Democrat-majority Houses (101st-103rd) and 


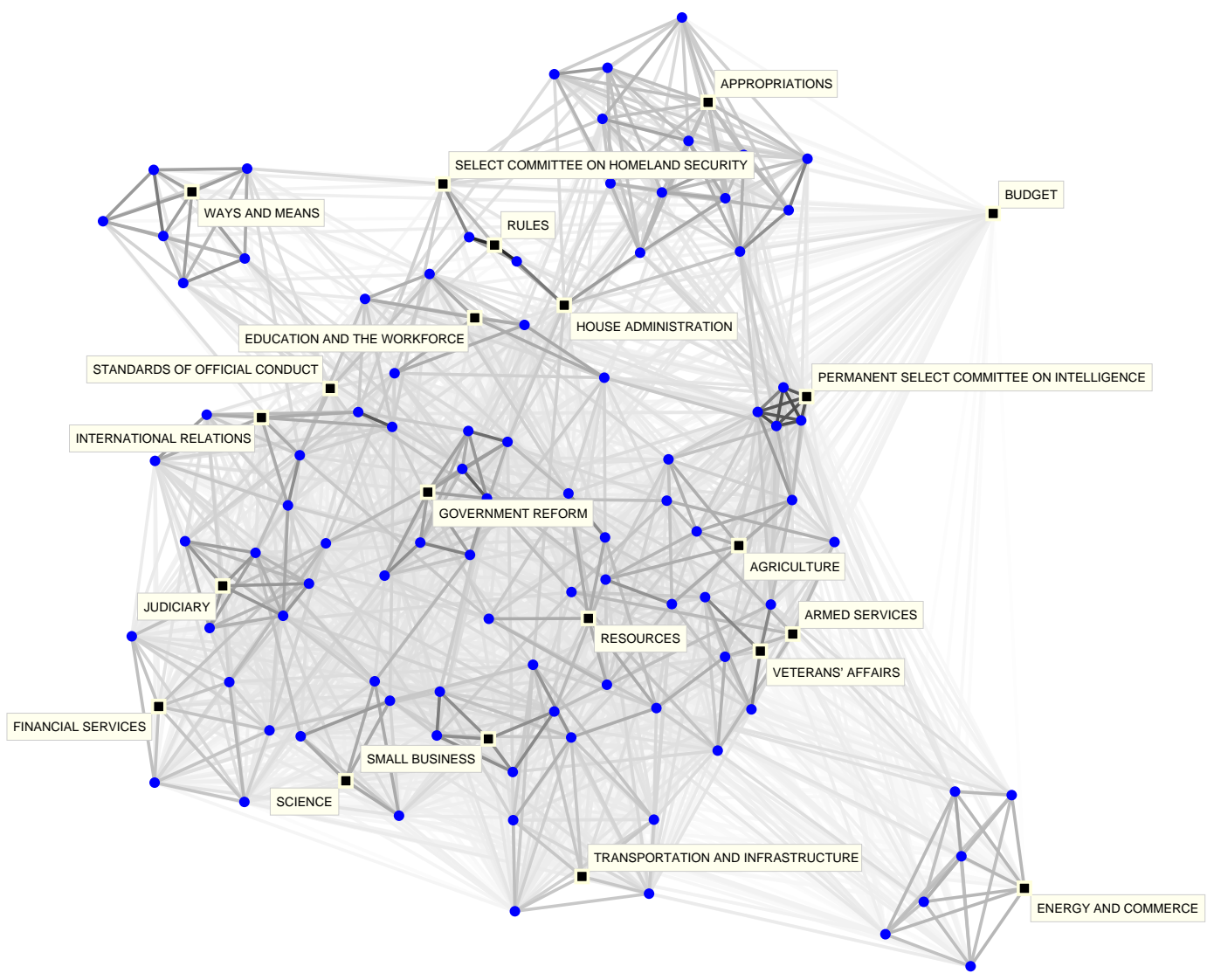

FIG. 2: (Color online) Network of committees (squares) and subcommittees (circles) in the 107th U.S. House of Representatives, with standing and select committees labeled. (Subcommittees tend to be closely tied to their main committee and are therefore left unlabeled.) Each link between two (sub)committees is assigned a strength (indicated by the link's darkness) equal to the normalized interlock. (The "interlock" between two committees is equal to the number of their common members. The normalization takes committee sizes into account by dividing the raw interlock by the expected number of common members if assignments were determined independently and uniformly at random.) Thus, lines between pairs of circles or pairs of squares represent normalized degree of joint membership between (sub)committees (it is because of this normalization that lines between squares are typically very light), and lines between squares and circles represent the fraction of standing committee members on subcommittees. This figure is drawn using a variant of the Kamada-Kawai spring-embedding visualization, which takes link strengths into account [25]. 


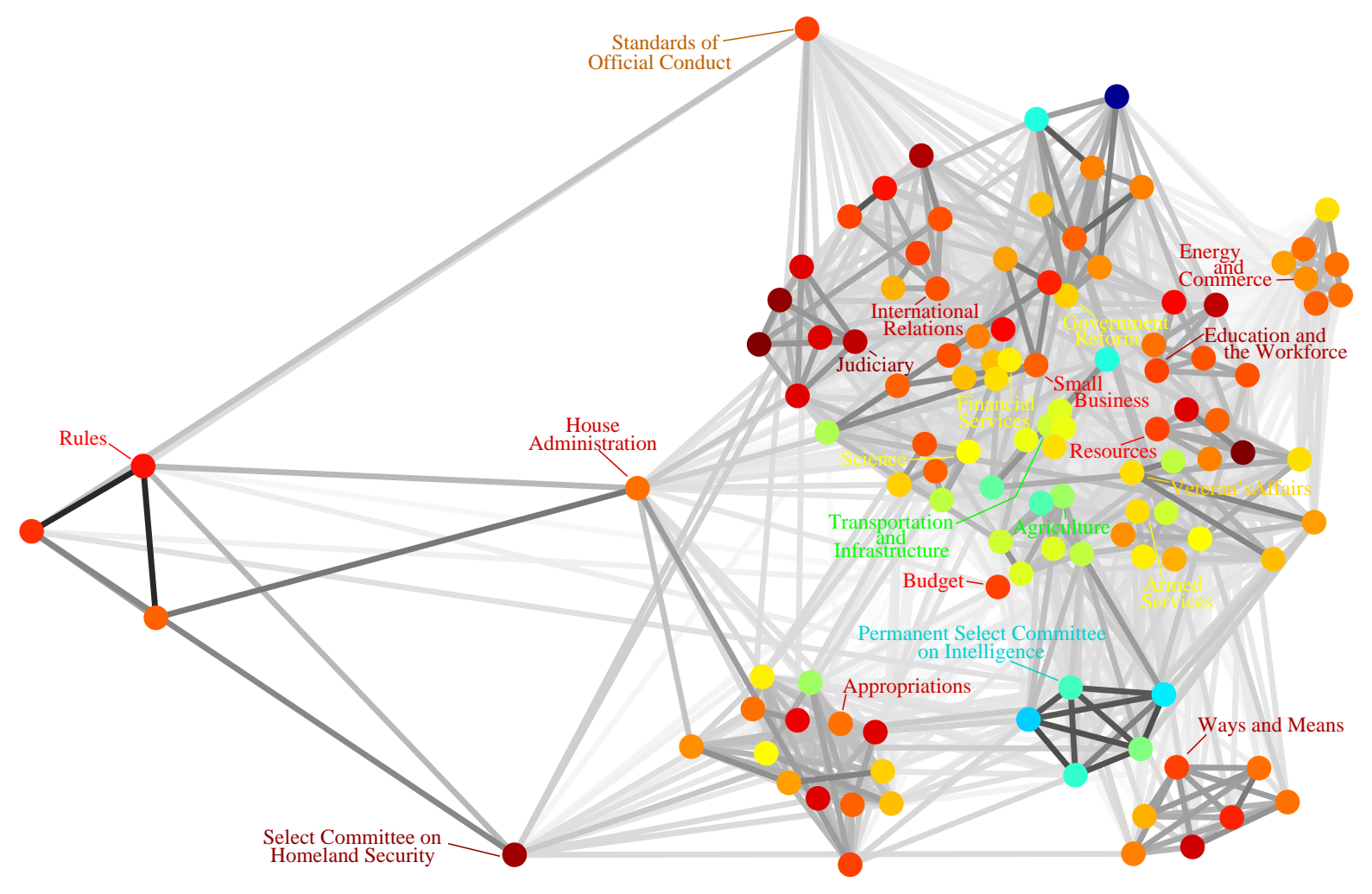

FIG. 3: (Color) Another visualization of the committee assignment network, with standing committees labeled. This plot is color-coded according to the mean "extremism" (defined in the text; see Appendix A for details) of each committee's constituents, where red nodes are more extreme and blue ones are less extreme. Thus, the redder committee are composed of ideologues from both parties and the bluer ones consist of moderates. The strength (darkness) of the link between two committees is equal to their interlock (which is not normalized here). Observe that similar groups of committees are clustered together in this plot as in Fig. 2, despite the different definition of link strengths. (This figure is also drawn using a variant of the Kamada-Kawai spring embedder.)

the Republican-majority ones (104th-108th)? As one means of addressing this question, we compute the degree of each node, defined as the number of edges connected to it. Because the committee assignment networks are bipartite, we construct two types of cumulative (integrated) degree distributions [40] and examine how they changed across Congresses. One distribution (Fig. 1a) indicates the number of committees on which each Representative 


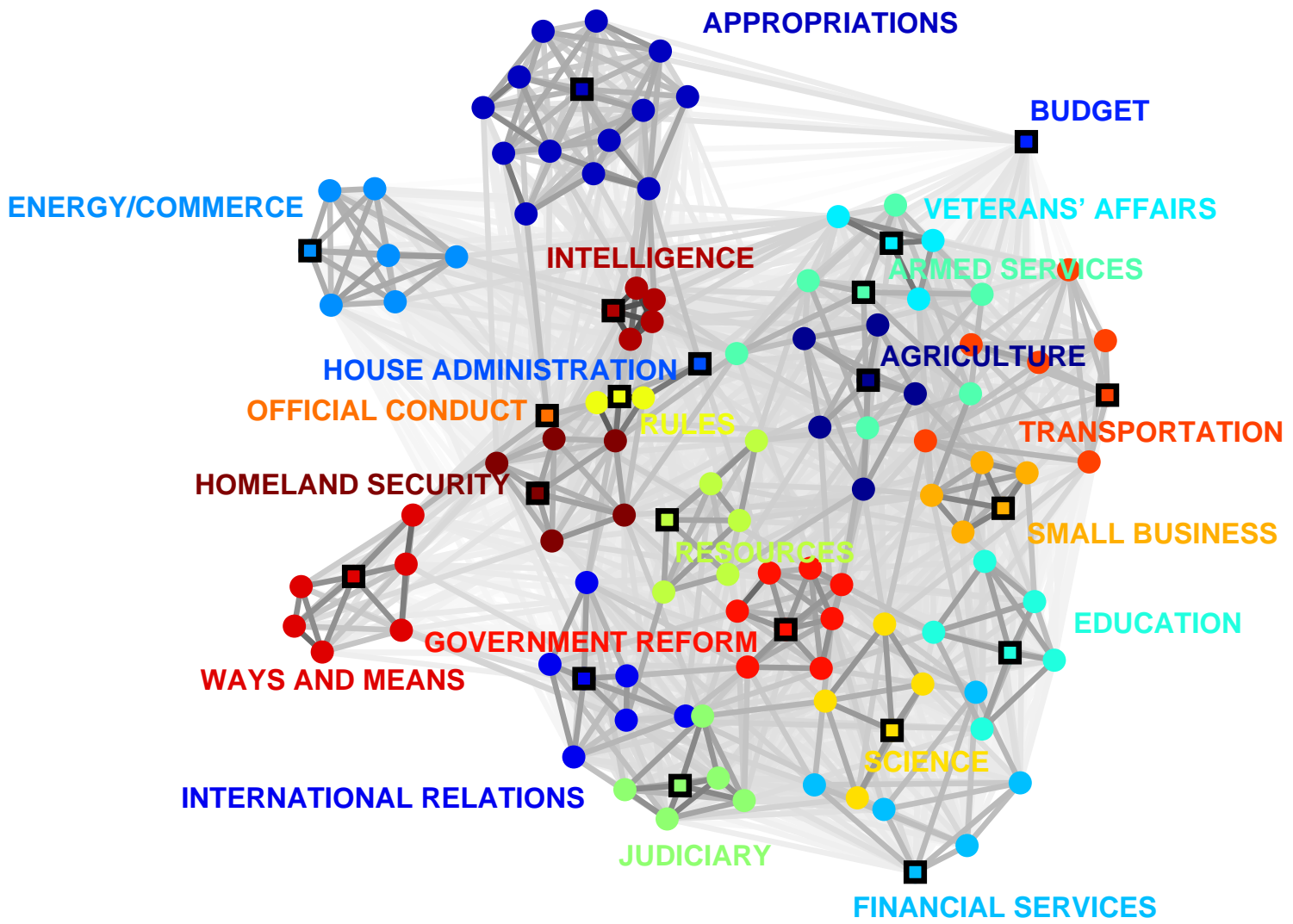

FIG. 4: (Color) Network of committees (squares) and subcommittees (circles) in the 108th U.S. House of Representatives, color-coded by the parent standing and select committees. (The depicted labels indicate the parent committee of each group but do not identify the location of that committee in the plot.) As with Fig. 2, this visualization was produced using a variant of the Kamada-Kawai spring embedder, with link strengths (again indicated by darkness) determined by normalized interlocks. Observe again that subcommittees of the same parent committee are closely connected to each other.

sits, and the other (Fig. 1b) gives the number of Representatives on each committee. We do not observe a significant trend in Democrat-majority Houses, although a slow increase in committee sizes is revealed in Fig. 1b. The committee reorganization that accompanied the formation of the Republican-majority 104th House, however, produced a sharp decline in the typical numbers of committee and subcommittee assignments per Representative, but the trend in subsequent Republican-majority Congresses has been a slow increase in both 


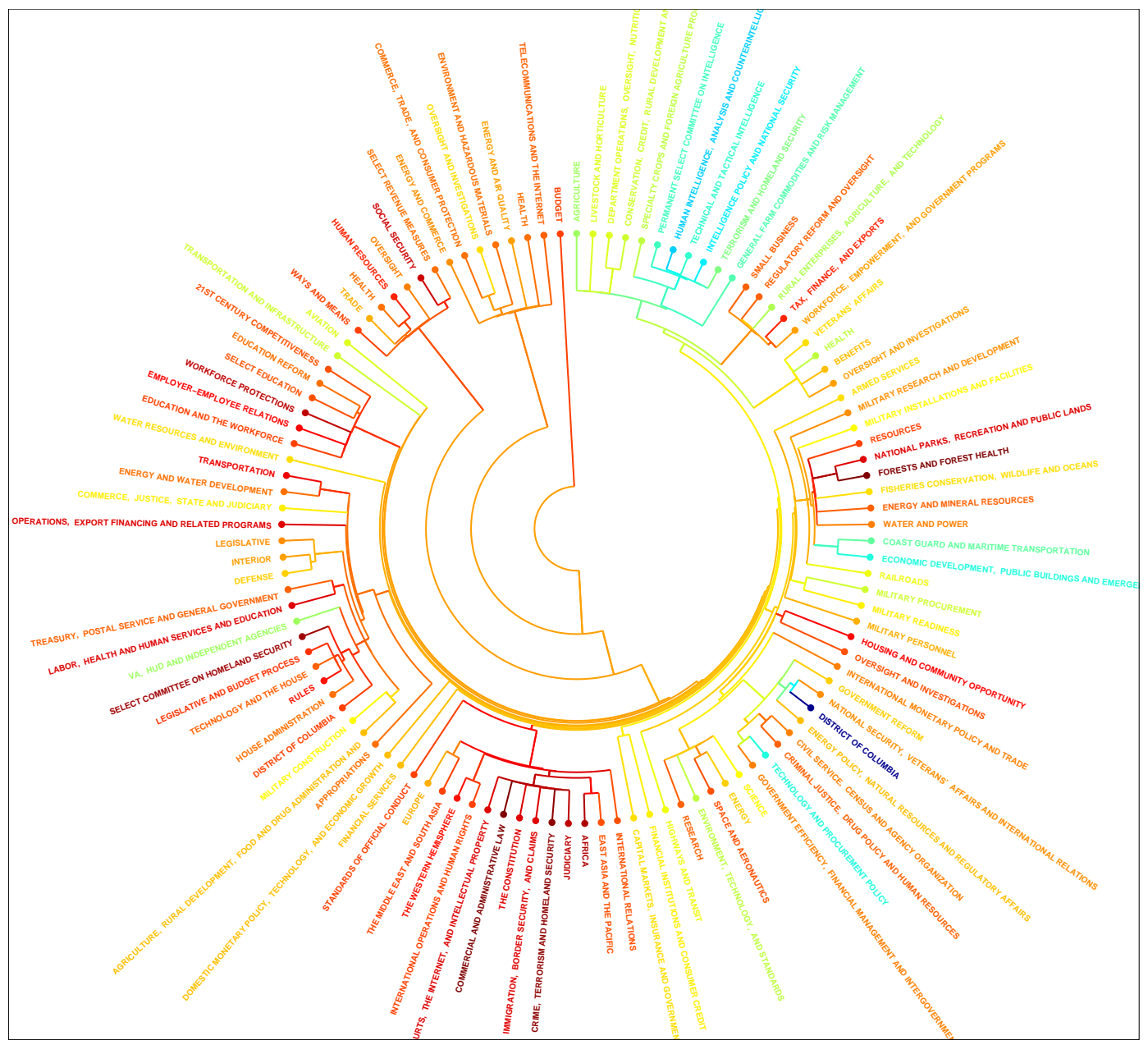

FIG. 5: (Color) Dendrogram representing the hierarchical clustering of the committees in the 107th U.S. House of Representatives, determined by single linkage clustering of normalized committee interlocks. Each committee is color-coded according to the mean "extremism" of its members (defined in the main text; see Appendix A), from less extreme (blue) to more extreme (red). The clusters at each level are color-coded according to the average of their constituent committee extremism scores.

the numbers of assignments and the committee sizes. These trends are visible in Fig. 1,

While rich in their data content, the two-mode networks of committee assignments are difficult to visualize and interpret. A common strategy in such cases is to examine instead a one-mode "projection" of the network onto either the committees or the Representatives. In our studies, we have made considerable use of the projection onto the committees, in which a network is created whose nodes represent the committees and whose edges represent 
common membership or "interlocks" between committees. Figures 2 and 3 show two different visualizations of the network of committees for the 107th House of Representatives (20012002), an example that we analyze in some depth below.

We quantify the strength of a connection between committees in this projected network with a normalized interlock, defined as the number of common members two committees have divided by the expected number of common members if membership of committees of the same size were randomly and independently chosen from Congressmen in the House. Committees with as many common members as expected by chance have normalized interlock 1 , those with twice as many have interlock 2 , those with none have interlock 0 , and so forth. We use this weighting in the visualization of the network of the 107th House in Fig. 2 by darkening the links between nodes accordingly [61]. As a comparison, we also show a visualization based on the raw (unnormalized) interlock count of common members in Fig. 3. In Fig. 4, we depict the 108th House using normalized interlock.

Some of the connections depicted in Figs. 24 are unsurprising. For instance, sets of subcommittees of the same standing committee typically share many of the same members, thereby forming a group or clique in the network. The four subcommittees of the 107th Permanent Select Committee on Intelligence, for example, each include at least half of the full 20-member committee and at least one third of each of the other subcommittees. These tight connections result in normalized interlocks with values in the range 14.4-23.6, causing these five nodes to be drawn close together in the visualizations, forming the small pentagon in the middle right of Fig. 2 and lower right of Fig. 3. The Intelligence Committee and its subcommittees are also tightly connected in the 108th House, appearing again as a small pentagon in Fig. 4 .

Some connections between committees, however, are less obvious. For instance, the 9member Select Committee on Homeland Security, formed in June 2002 during the 107th Congress in the aftermath of the terrorist attacks of September 11, 2001 [26], has a strong connection to the 13-member Rules Committee (with a normalized interlock of 7.4 from two common members), which is the committee charged with deciding the rules and order of business under which legislation is considered by other committees and the full House [56]. The Homeland Security Committee is also connected to the 7-member Legislative and Budget Process Subcommittee of Rules by the same two common members (with normalized interlock 13.7). In the 108th Congress (see Fig. 4), the Homeland Security Committee 
swelled to 50 members but maintained a close association with the Rules Committee (with a normalized interlock of 4.1 from 6 common members).

\section{THE HIERARCHICAL STRUCTURE OF COMMITTEES}

We now turn to an examination of community structure in the networks of committees based on the one-mode projection described above. We do this using several methods of hierarchical clustering, in which one begins with a network and ends up (by construction) with a hierarchical (tree) structure. In this section, we discuss the hierarchical clustering method known as single linkage clustering [24]. We found similar results using several alternative community detection methods, which are discussed in detail in Appendix B. For each of these methods, we quantify the organizational structures we find using HortonStrahler numbers (to indicate the number of hierarchical levels) and modularity (to indicate the compartmentalization into different groups).

To implement single linkage clustering, we start with the complete set of committees for a given Congress. We then join committees sequentially starting with the pair with the greatest normalized interlock, followed by the next greatest, and so forth. This process generates "clusters" of committees, which can be represented using a tree or dendrogram, such as that shown in Fig. 5 for the 107th House. Closer examination of the dendrograms in Fig. 5 (from the 107th Congress) and Fig. 6 (from the 108th Congress), each of which has a Horton-Strahler number of 5, conveys four reasonably-expected and well-ordered hierarchical levels of clustering: subcommittees, standing and select committees, groups of standing and select committees, and the entire House. These single linkage clustering dendrograms also give some suggestion of a weaker fifth level of organization corresponding to groups of subcommittees inside larger standing committees.

In this paper, we are primarily interested in the organizational levels describing the connections between standing and select committees (and groups thereof). For example, near the 8 o'clock position in Fig. 5 (from the 107th Congress) is a tightly grouped cluster that includes the House Rules Committee, the House Administration Committee, and the Select Committee on Homeland Security. A similar cluster appears in Fig. 6 (from the 108th Congress) near the 3 o'clock position, including all five of the subcommittees of Homeland Security introduced in the 108th Congress. Because assignments to select committees 


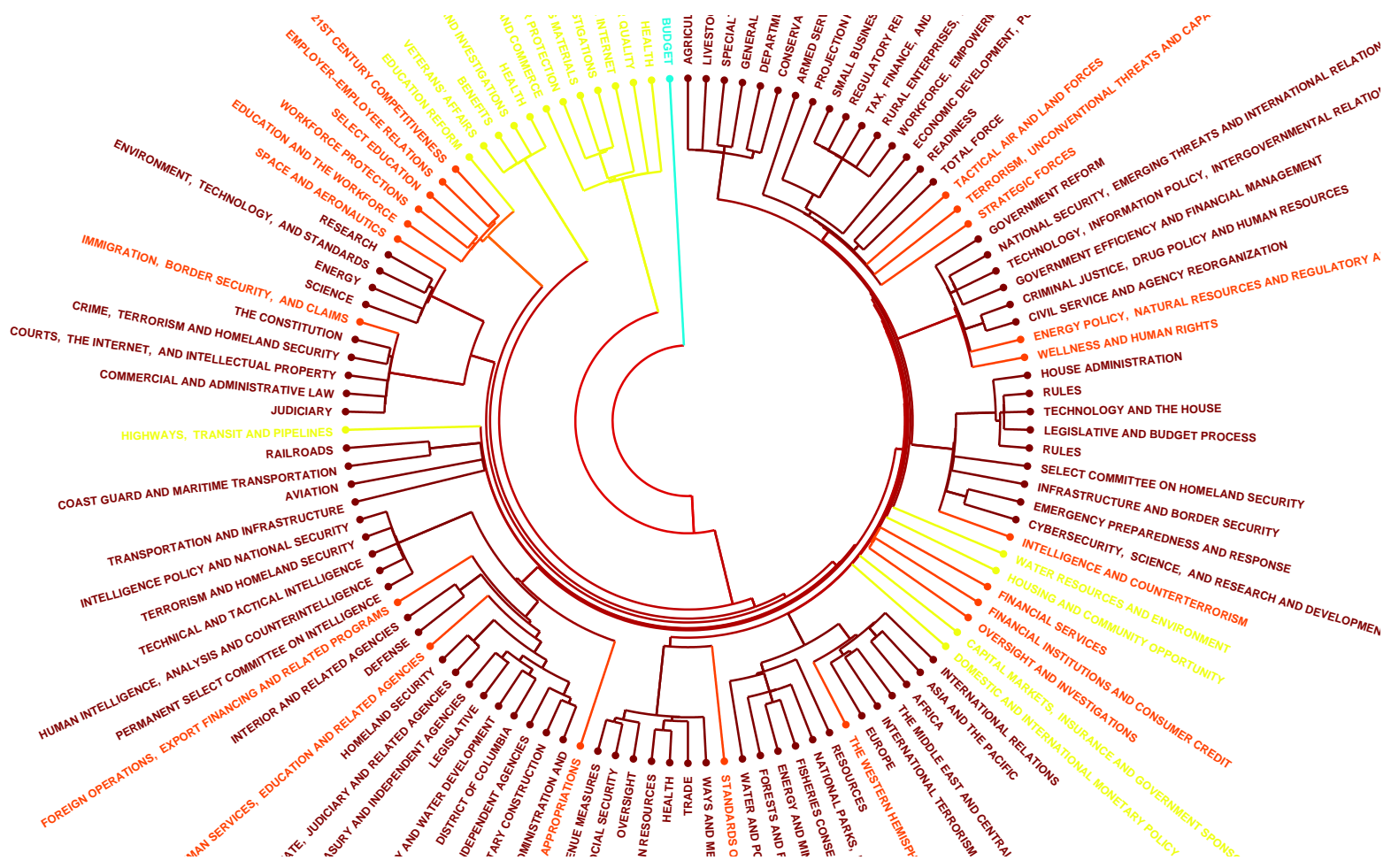

FIG. 6: (Color) Dendrogram of the 108th House, determined by single linkage clustering and colorcoded according to the Horton-Strahler values [22, 54] of its leaves (discussed in the text), with lower values in blue and higher values in red. The ties between Rules and Homeland Security persist (between the 3 o'clock and 4 o'clock positions), despite the swelling in size of the latter committee to fifty members. As discussed in Appendix B, these ties are robust with respect to the algorithm used to determine the hierarchy.

are ordinarily determined by drawing selectively from committees and subcommittees with overlapping jurisdiction, one might naively expect a close connection between the Select Committee on Homeland Security and, for example, the Terrorism and Homeland Security Subcommittee of the Intelligence Select Committee, which was formed originally as a bipartisan "working group" and was designated on September 13, 2001 by Speaker of the House Dennis Hastert [R-IL] as the lead congressional entity assigned to investigate the 9/11 terrorist attacks [26]. However, the 107th Homeland Security Committee shares only one common member (normalized interlock 2.4) with the Intelligence Select Committee (located near the 1 o'clock position in Fig. 5) and has no interlock at all with any of the four Intelligence subcommittees.

As shown in Figure 5, we can enrich the analysis by color-coding each committee according 
to the mean "extremism" of its members. Extremism is determined using the results of a singular value decomposition analysis of Representatives' voting records that places each Representative on a scale that runs, roughly speaking, from the most partisan Republican members of the House to the most partisan Democrats (the SVD analysis is described in detail in Appendix A). The extremism of a committee is then quantified as the average deviation of its members from the mean on this scale. Committees composed of highly partisan members of either party appear in red in Fig. 5 and those containing more moderate Representatives appear in blue. Taking again the examples of Intelligence and Homeland Security, we can immediately identify the former as moderate and the latter as more partisan. Indeed, the Select Committee on Homeland Security has a larger mean extremism than any of the 19 standing Committees and has the 4th largest mean extremism among the 113 committees of the 107th House (see Table I). This is perhaps not so surprising when we see that its members included the House Majority Leader, Richard Armey [R-TX], and both the Majority and Minority Whips, Tom DeLay [R-TX] and Nancy Pelosi [D-CA]. However, our characterization of the committee was made mathematically, using no political knowledge beyond the roll call votes of the 107th House. As another example, the 107th House Rules Committee is the second most extreme of the 19 standing committees (after Judiciary) and ranks 18th out of 113 committees overall. In contrast, the Permanent Select Committee on Intelligence of the 107th House has a smaller mean extremism than each of the 19 standing Committees, and Intelligence and its four subcommittees all rank among the 10 least extreme of all 113 committees.

\section{MODULARITY}

To further investigate the observed hierarchies in the House committee assignment networks, we employ the statistic known as modularity, modified to allow for the weighted nature of our networks. Consider first an unweighted network, which is divided into some number of groups of vertices. The modularity $m$ for this division into groups is defined to be [35]

$$
m=\sum_{i}\left(e_{i i}-a_{i}^{2}\right)
$$

where $e_{i j}$ denotes the fraction of ends of edges in group $i$ for which the other end of the edge lies in group $j$ and $a_{i}=\sum_{j} e_{i j}$ is the fraction of all ends of edges that lie in group $i$. 


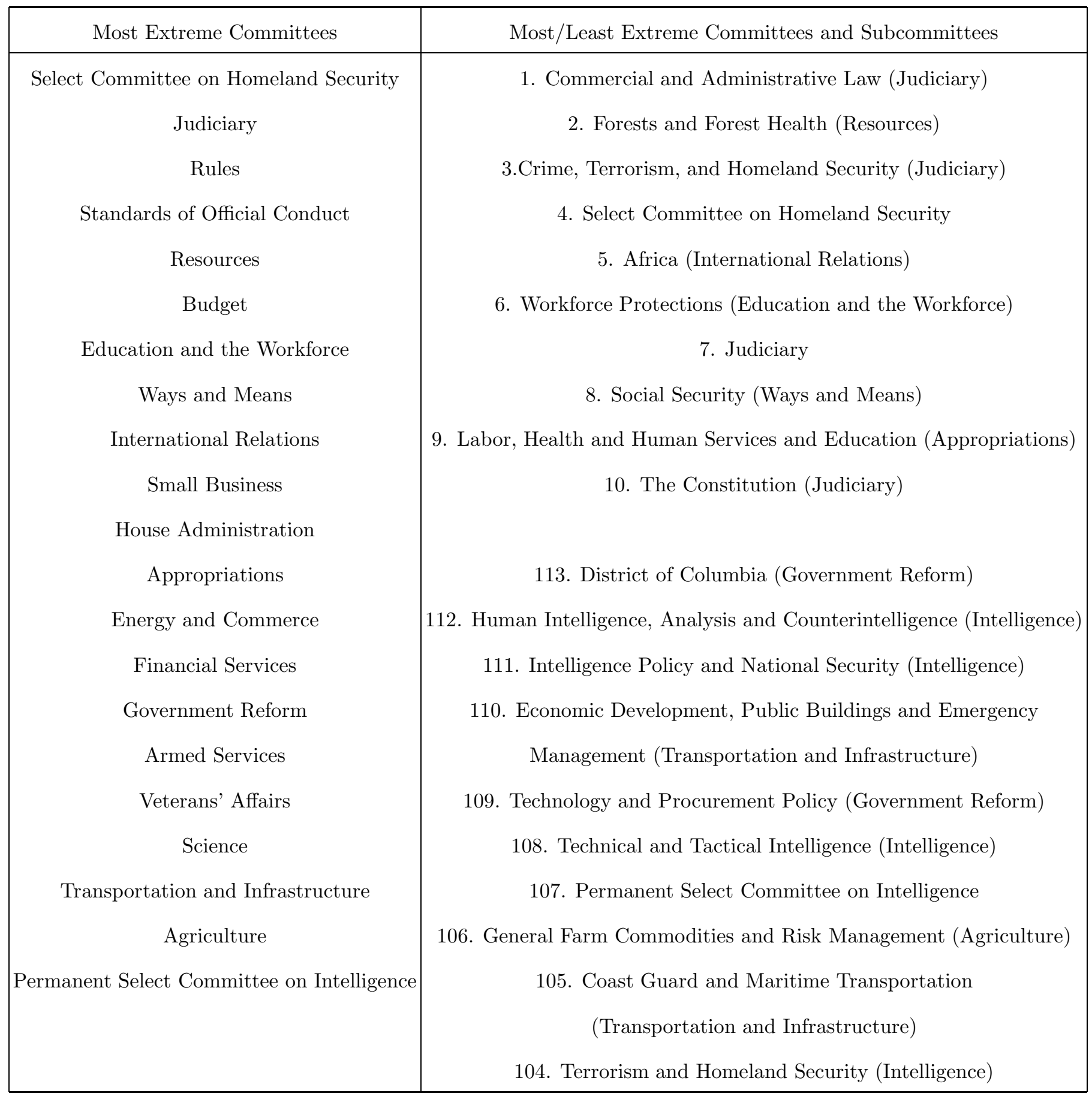

TABLE I: SVD rank ordering of the committees in the 107th House. In the first column, we list the standing and select committees from most extreme to least extreme. In the second column, we list the most extreme and least extreme committees and subcommittees, with the parent committee shown in parentheses when appropriate. (The latter set of committees is listed from less extreme to more extreme.) 
Modularity measures the difference between the total fraction of edges that fall withinrather than between-groups (the first term) and the fraction one would expect if edges were placed at random (respecting vertex degrees). Thus, high values of the modularity indicate partitions of the network in which more of the edges fall within groups than one would expect by chance. This, in turn, has been found to be a good indicator of functional network divisions in many cases [37].

The projected one-mode networks we consider here are weighted. In our calculations, we therefore employ the weighted generalization of modularity described in [34], in which instead of counting numbers of edges falling between particular groups, we count the sums of the weights of those edges, so that heavily weighted edges contribute more than lightly weighted ones. Both $e_{i j}$ and $a_{i}$ can be generalized in this fashion in a straightforward manner, and then the modularity is again calculated from Eq. (1). The meaning of the modularity remains essentially the same: it measures when a particular division of the network has more edge weight within groups than one would expect on the basis of chance.

We use modularity to quantify the organizational divisions of the networks and to compare the dendrograms to each other. In particular, the modularity values shown in Table Indicate that the dendrograms produced via single linkage clustering have a better-defined community structure (higher modularity) in the Republican-controlled Houses (104th-108th) than in the Democrat-controlled ones. Hence, with respect to the metric of normalized interlock, the committee reorganization following the Republican Revolution (which we already saw in Fig. 19 produced a sharp decline in the typical numbers of committee assignments per Representative compared to the 101st-103rd Houses) seems also to have tightened the compartmentalization of the House committee assignments.

\section{NUMBER OF HIERARCHICAL LEVELS}

Another interesting feature of dendrograms is the depth of their hierarchical organization, which can be quantified by computing Horton-Strahler numbers [2, 10, 22, 54]. 62] As originally defined, Horton-Strahler numbers give the number of levels in the minimum-depth branch of a tree. Here we consider two generalizations. First, we examine the Strahler

numbers of leaves (see, for example, Fig. 6), assigning a value $S_{j}$ that identifies the number of hierarchical levels associated with the $j$ th subcommittee; this is the number of levels in 


\begin{tabular}{|c|c|c|c|c|}
\hline Method: & $\mathrm{SL}$ & $\mathrm{EB}$ & $\mathrm{NB}$ & $\mathrm{WL}$ \\
\hline$M_{1}, \tilde{M}_{1}$ & $0.1666,0.1037$ & $0.1450,0.0904$ & $0.1775,0.0964$ & $0.3388,0.1945$ \\
\hline$M_{2}, \tilde{M}_{2}$ & $0.1831,0.0923$ & $0.1552,0.0855$ & $0.1693,0.0979$ & $0.3395,0.1675$ \\
\hline$M_{3}, \tilde{M}_{3}$ & $0.1824,0.0975$ & $0.2640,0.1388$ & $0.2385,0.1308$ & $0.3884,0.2343$ \\
\hline$M_{4}, \tilde{M}_{4}$ & $0.4221,0.2077$ & $0.2987,0.1542$ & $0.3312,0.1909$ & $0.4432,0.2614$ \\
\hline$M_{5}, \tilde{M}_{5}$ & $0.3331,0.1681$ & $0.2518,0.1290$ & $0.2439,0.1600$ & $0.3945,0.2228$ \\
\hline$M_{6}, \tilde{M}_{6}$ & $0.2982,0.1709$ & $0.2481,0.1305$ & $0.2420,0.1581$ & $0.3720,0.1861$ \\
\hline$M_{7}, \tilde{M}_{7}$ & $0.3350,0.1755$ & $0.2604,0.1293$ & $0.2386,0.1465$ & $0.3748,0.2133$ \\
\hline$M_{8}, \tilde{M}_{8}$ & $0.3324,0.1736$ & $0.2319,0.1178$ & $0.2294,0.1417$ & $0.3781,0.2204$ \\
\hline
\end{tabular}

TABLE II: Maximum $\left(M_{i}\right)$ and average $\left(\tilde{M}_{i}\right)$ modularities of community structure for committee assignment networks for the 101st-108th House of Representatives, with dendrograms produced using single linkage clustering (SL), random-walk betweenness with sequential edge (committee assignment) removal (EB), random-walk betweenness with sequential Representative node removal (NB), and a generalization of the local community detection algorithm of [3] to weighted networks (WL). These last three algorithms are described in Appendix B. For the WL method, we use the value of the local clustering threshold parameter $\alpha$ giving the greatest maximum modularity (see Appendix B).

the particular branch of the tree with that specific subcommittee as the leaf. In Table VIII of Appendix B, we compare $S=\max _{j} S_{j}$, the mean $\tilde{S}=\left\langle S_{j}\right\rangle$, and the standard deviation $\sigma=\left\langle\left(S_{j}-\tilde{S}\right)^{2}\right\rangle^{1 / 2}$ in the 101st-108th Houses for single linkage clustering, two betweennessbased dendrograms, and our local community detection method to quantify the statistics of the hierarchical levels revealed by each method. Second, we also define a notion of Strahler numbers for communities (see Fig. 7), in which a given subtree (i.e., community) is assigned a Strahler number as if it were itself a full dendrogram.

As indicated previously, single linkage clustering identifies several hierarchical levels of organization within the House of Representatives: subcommittees, committees, and the floor (the whole House). In all eight Houses, we also identify a fourth hierarchical level, representing groups of closely-connected committees. In the 104th, 105th, 107th, and 108th Houses, single linkage clustering reveals a fifth level of organization. See, for example, Fig. 6, which is color-coded according to the $S_{j}$ values of the leaves/committees, and Fig. 7, which is 
color-coded according to the Horton-Strahler $S$ values computed as if each community were itself an individual tree. Single linkage clustering appears to organize the House's hierarchical structure more sharply than betweenness-based dendrograms, as the trees produced by the former have consistently higher values of $S$ and $\tilde{S}$. Additionally, networks with high maximum and average Strahler numbers tend also to have high modularity scores (compare Tables II and VIII), signifying their strong organizational structure.

Strahler numbers reveal additional information about the changes in the House committee assignment networks after the Republican Revolution. The three lowest mean Strahler values occur in the Democrat-majority Houses (101st-103rd), despite the fact that the number of committees and subcommittees decreased after the Republicans gained control of the House (see Fig. 1). In a perfectly balanced binary tree, one would instead expect an increase in the Strahler number when nodes are added. Furthermore, all of the Republican-controlled Houses except the 106th have five levels of hierarchical structure (based on the metric of Strahler numbers) rather than the four revealed in the 101st-103rd Houses, so it seems that the change in majority party added an extra level of hierarchical structure to the committee assignment network.

\section{CONCLUSIONS}

We have applied methods from network theory, coupled with an SVD analysis of roll call votes, to investigate the organizational structure of the committees and subcommittees of the U.S. House of Representatives. Using the 101st-108th Congresses as examples, we have found evidence of several levels of hierarchy within the network of committees and - without incorporating any knowledge of political events or positions - identified some close connections between committees, such as that between the House Rules Committee and the Select Committee on Homeland Security in the 107th and 108th Congresses. We have also identified correlations between committee assignments and Representatives' political positions and examined changes in the network structure across different Congresses, emphasizing effects that resulted from the shift in majority control from Democrats to Republicans starting with the 104th House. 


\section{Acknowledgements}

We thank Gordon Kingsley for challenging ideas that prompted some of this research; Michael Abraham for developing some of the computer codes; Casey Warmbrand for converting data into a usable format; Thomas Callaghan for his computer codes and comments on this manuscript; Ron Burt, Aaron Clauset, Sharad Goel, Debra Goldberg, Chris Wiggins, and Yan Zhang for useful conversations; and James Fowler for a critical reading of this manuscript. We thank two anonymous referees for critical comments that lead to changes that have improved the paper. We also acknowledge support provided by a National Science Foundation VIGRE grant awarded to the School of Mathematics at Georgia Tech. MAP was also supported in part by the Gordon and Betty Moore Foundation through Caltech's Center for the Physics of Information. PJM was also supported by start-up funds provided by the Institute for Advanced Materials, Nanoscience and Technology and the Department of Mathematics at the University of North Carolina at Chapel Hill. We obtained the roll call data for the 102nd-107th Congresses from the Voteview website [44], the roll call data for the 101st Congress from the Inter-University Consortium for Political and Social Research [58], and the committee assignments for the 101st-108th Congresses from the web site of the House of Representatives Office of the Clerk [57].

\section{APPENDIX A: VOTING PATTERNS}

In this appendix, we analyze the voting records of Representatives. The results of this analysis were used in the main text to investigate the relationship between the network of interlocks linking the Congressional committees and the political positions of their constituent Representatives.

One way to characterize political positions is to tabulate individuals' voting records on selected key issues (via, for example, interest group ratings), but such a method is subjective by nature and a procedure that involves less personal judgment is preferable. Here we apply the "multi-dimensional scaling" technique known as singular value decomposition (SVD) [18, 53] to the complete voting records of each session of the House [45, 46]. Each two-year term

of Congress is treated in isolation from the others. Other methods of analysis [23, 42], such as the Bayesian approach of [8], also yield useful results. The advantages of multi-dimensional 
scaling techniques versus factor analysis (which has a long tradition in political science) in analyzing voting data are discussed in detail in [5].

We define an $n \times m$ voting matrix $\mathbf{B}$ with one row for each of the $n$ Representatives in the House and one column for each of the $m$ votes taken during a two-year term. For instance, the 107th House had $n=444$ Representatives (including midterm replacements) and took $m=990$ roll call votes. The element $B_{i j}$ is +1 if Representative $i$ voted "yea" on measure $j$ and -1 if he or she voted "nay." If a Representative did not vote because of absence or abstention, the corresponding element is 0 . (We do not separately identify abstentions from absences; additionally, a relatively low number of false zeroes is generated by resignations and midterm replacements.)

The SVD identifies groups of Representatives who voted in a similar fashion on many measures. The grouping that has the largest mean-square overlap with the actual groups voting for or against each measure is given by the leading (normalized) eigenvector $\mathbf{u}^{(1)}$ of the matrix $\mathbf{B}^{T} \mathbf{B}$, the next largest by the second eigenvector $\mathbf{u}^{(2)}$, and so on [18, 53]. If we denote by $\sigma_{k}^{2}$ the corresponding eigenvalues (which are provably non-negative) and by $\mathbf{v}^{(k)}$ the normalized eigenvectors of $\mathbf{B B}^{T}$ (which have the same eigenvalues), then it can be shown that

$$
B_{i j}=\sum_{k=1}^{n} \sigma_{k} u_{i}^{(k)} v_{j}^{(k)},
$$

where $\sigma_{k} \geq 0$ for all $k$. The matrix $\mathbf{B}^{(r)}, r<n$, with elements

$$
B_{i j}^{(r)}=\sum_{k=1}^{r} \sigma_{k} u_{i}^{(k)} v_{j}^{(k)}
$$

approximates the full voting matrix $\mathbf{B}$. The sum of the squares of the errors in the elements is equal to $\sum_{k=r+1}^{n} \sigma_{k}^{2}$, which vanishes in the limit $r \rightarrow n$. Assuming the quantities $\sigma_{k}$, called the singular values, are ordered such that $\sigma_{1} \geq \sigma_{2} \geq \sigma_{3} \ldots$, this implies that $\mathbf{B}^{(r)}$ is a good approximation to the original voting matrix provided the singular values decay sufficiently rapidly with increasing $k$. Alternatively, one can say that the $l$ th term in the singular value decomposition (A1) accounts for a fraction $\sigma_{l}^{2} / \sum_{k=1}^{n} \sigma_{k}^{2}$ of the sum of the squares of the elements in the voting matrix.

To an excellent approximation, we find that a Representative's voting record can be characterized by just two coordinates. That is, $B_{i j}^{(2)}$ is a good approximation to $B_{i j}$. Observing that one of the two directions correlates well with party affiliation for members of the two 
major parties, we call this the "partisan" coordinate. We call the other direction the "bipartisan" coordinate, as it correlates well with how often a Representative votes with the majority. Because Senators are generally better known than Representatives, we plot as an example the coordinates along these first two eigenvectors for the 107th Senate in Fig. 87. As expected, Democrats are grouped together and are almost completely separated from Republicans. For ease of identification, we follow the sign convention that places Democrats on the left and Republicans on the right. The few instances of apparent party misidentification by the partisan coordinate in Fig. 8 are unsurprising. Conservative Democrats, such as Zell Miller [D-GA], appear farther to the right than some moderate Republicans [4]. Additionally, Senator James Jeffords [I-VT], who left the Republican party to become an Independent early in the 107th Congress, appears closer to the Democratic group than the Republican one and to the left of several of the more conservative Democrats. (He appears twice in Fig. 8a, once each for votes cast under his two different affiliations.)

One can use a truncation of the SVD to construct an approximation to the votes in the full roll call [53]. For instance, with our two-dimensional approximation to the voting matrix, we assign "yea" or "nay" votes to individuals based on the signs of the corresponding elements of the matrix. In Fig. $8 \mathrm{~b}$, we show the fraction of actual votes correctly reconstructed by this approximation, which gives a measure of the "predictability" of the Senators in the 107th Congress. For both parties, moderate Senators are less predictable than hardliners. The two-dimensional projection correctly reconstructs the votes of some hard-line Senators for as many as $97 \%$ of the votes they cast. Examining the apparent outliers in Fig. 8b, the votes Senator Jeffords cast as a Republican appear here to make him the least "predictable" Senator. However, it is important to emphasize that Jeffords cast relatively few votes as a member of the Republican party, so it is not surprising that this behavior is less predictable because the voting record includes a large number of artificial absences. The other Independent in Fig. 8b is Senator Dean Barkley [I-MN], who completed the remainder of the term for Senator Paul Wellstone [D-MN] in the 107th Congress after Wellstone's death. While one might be tempted to interpret Barkley's partisan coordinate as balanced, its value is strongly influenced by the large number of effective absences in the SVD analysis because he was not appointed until very late in the term. Both of his first two coordinates consequently lie near zero. The other apparent outliers in Fig. 8b-Senators Russ Feingold [D-WI] and John McCain [R-AZ] - are both known for their occasional "maverick" behavior. 


\begin{tabular}{|c|c|c|c|c|c|c|}
\hline Congress & 1 st & 2nd & $3 \mathrm{rd}$ & 4 th & 5 th & 6 th \\
\hline $101 \mathrm{st}$ & 40.0 & 20.4 & 3.0 & 1.7 & 1.5 & 1.1 \\
\hline $102 \mathrm{nd}$ & 39.6 & 20.1 & 2.8 & 1.8 & 1.2 & 1.0 \\
\hline $103 \mathrm{rd}$ & 43.1 & 21.5 & 2.9 & 1.6 & 1.3 & 0.9 \\
\hline $104 \mathrm{th}$ & 47.1 & 19.1 & 2.7 & 1.6 & 1.3 & 0.7 \\
\hline $105 \mathrm{th}$ & 38.6 & 28.7 & 2.0 & 1.5 & 1.2 & 0.9 \\
\hline $106 \mathrm{th}$ & 40.0 & 29.5 & 1.9 & 1.6 & 1.2 & 0.9 \\
\hline $107 \mathrm{th}$ & 45.3 & 29.6 & 1.6 & 1.4 & 1.0 & 0.7 \\
\hline
\end{tabular}

TABLE III: Amount of voting information, measured as the percentage of the variance of the voting matrix, encoded by the six leading eigenvectors of the 101st-107th House voting matrices. The first column indicates the Congress, and the next six columns give the percentage of information encoded by each of the six leading eigenvectors.

Having demonstrated the application of SVD to the analysis of the voting records of the Senate, let us now return to the House of Representatives. For the 107th House, we find that the leading eigenvector accounts for about $45.3 \%$ of the variance of the voting matrix, the second eigenvector accounts for about 29.6\%, and no other eigenvector accounts for more than 1.6\%. We obtain similar results for other recent Congresses, with two eigenvectors giving a good approximation to the voting matrix in every case (see Table III). In Fig. 9, we plot these two coordinates for every member of the House of Representatives for each of the 102nd-107th Congresses. It has been shown previously using other methods that Congressional voting positions are well-approximated by just two coordinates [44, 45], but it is important to emphasize that different identification methods treat the "bipartisan" direction differently. In particular, some methods eliminate it entirely and associate the two remaining dimensions with partisanship and an additional direction often identified as a North-South axis, which was historically important during periods of heightened concern about civil rights [44, 45]. The SVD analysis here keeps the "bipartisan" coordinate, making identifications in a particularly simple fashion straight from the voting matrix containing the roll call data.

As with the Senate, we find that the leading eigenvector corresponds closely to the acknowledged political party affiliation of the Representatives, with Democrats (blue) on the 
left and Republicans (red) on the right in the plots [63]. Representatives who score highly on this "partisan" coordinate - either positively or negatively - tend often to vote with members of their own party. From this coordinate, we also compute a measure of "extremism" for each Representative as the absolute value of their partisan coordinate relative to the mean partisan score of the full House. That is, we define the extremism $e_{i}$ of a Representative by $e_{i}=\left|p_{i}-\mu\right|$, where $p_{i}$ is the Representative's partisanship score and $\mu$ is the mean value (usually skewed slightly towards the majority party) of that coordinate for the entire House. In Table IV], we list the most and least partisan Representatives from each party (as computed from the roll call) for the 107th House.

By contrast, the second eigenvector groups essentially all Representatives together regardless of party affiliation and thus appears to represent voting actions in which most members of the House either approve or disapprove of a motion simultaneously. Representatives who score highly on this "bipartisan coordinate" tend to often vote with the majority of the House.

The mean extremism for the Representatives in the 107th House is about 0.0458, and the standard deviation is $\sigma \simeq 0.0090$. The extremism of committees as averages over their constituent members yields a distribution of mean 0.0456 and standard deviation 0.0032 . By contrast, one might crudely expect the standard deviation of the committees' extremism to be approximately $\sigma / \sqrt{N}$, where $N$ is the average number of Representatives per committee. For the 107th House, this gives $0.0090 / \sqrt{21.87} \simeq 0.0019$. Hence, the distribution of committee extremism is roughly $50 \%$ wider than what would be expected with independent committee assignments. Basic statistics concerning committee extremism for the 101st107th Houses are summarized in Table V. Observe, for example, that the relative variance versus that expected from random committee and subcommittee assignments increases with every Congress (with the largest increase occurring between the 106th and 107th Houses).

Using the SVD results, we can also calculate the positions of the votes (as opposed to the voters) along the same two leading dimensions to quantify the nature of the issues being decided. We show this projection for the 107th House in Fig. 10. One application of this analysis is a measurement of the reproducibility of individual votes and outcomes. Reconstituting the voting matrix as before using only the information contained in the two leading singular values and the corresponding eigenvectors and summing the resulting approximated votes over all Representatives, we derive a single score for each vote. Making 


\begin{tabular}{|c|c|c|}
\hline Least Partisan & Farthest Left & Farthest Right \\
\hline K. Lucas [R] & J. D. Schakowsky [D] & T. G. Tancredo [R] \\
C. A. Morella [R] & J. P. McGovern [D] & J. B. Shadegg [R] \\
R. M. Hall [D] & H. L. Solis [D] & J. Ryun [R] \\
R. Shows [D] & L. C. Woolsey [D] & B. Schaffer [R] \\
G. Taylor [R] & J. F. Tierney [D] & P. Sessions [R] \\
C. W. Stenholm [D] & S. Farr [D] & S. Johnson [R] \\
R. E. Cramer [D] & N. Pelosi [D] & B. D. Kerns [R] \\
V. H. Goode [R] & E. J. Markey [D] & P. M. Crane [R] \\
C. John [D] & J. W. Olver [D] & W. T. Akin [R] \\
C. C. Peterson [D] & L. Roybal-Allard [D] & J. D. Hayworth [R] \\
\hline
\end{tabular}

TABLE IV: SVD rank ordering of the most and least partisan Representatives in the 107th U.S. House. The first column gives the least partisan Representatives, as determined by an SVD of the roll call votes. The second column gives the SVD rank ordering of the most partisan Representatives. They are all Democrats (the mean partisanship is skewed slightly towards the Republican party because it held the majority), so this also ranks the Representatives farthest to the Left. The third column gives the rank ordering of the Representatives farthest to the Right.

a simple assignment of "pass" to those votes that have a positive score and "fail" to all others successfully reconstructs the outcome of 984 of the 990 total votes (i.e., about $99.4 \%$ of them). Overall, 735 (about $74.2 \%$ ) of the votes passed, so simply guessing that every vote passed would be considerably less effective. Ignoring values from known absences and abstentions (i.e., zeroes in the original voting matrix), the analysis still identifies 975 of the 990 results correctly. Even with the most conservative measure of the reconstruction success rate -in which we ignore values associated with abstentions and absences, assign individual yeas or nays according to the sign of the matrix elements, and then observe which outcome has a majority in the resulting roll call-the two-dimensional reconstruction still identifies 939 (about 94.8\%) of the outcomes correctly. We repeated these calculations for the 101st-106th Houses and found similar results in each case (see Table VI). The remarkable accuracy of SVDs in reconstructing votes was previously observed for U.S. Supreme Court cases in [53]. The Optimal Classification (OC) technique of [46] (see also [44]) also generates 


\begin{tabular}{|c|c|c|c|c|c|c|c|}
\hline House & $\mu_{R}, \operatorname{Var}_{R}$ & $\mu_{C}, \operatorname{Var}_{C}$ & $\mu_{S}, \operatorname{Var}_{S}$ & $\langle C\rangle$ & $\Delta\left(\operatorname{Var}_{C}\right)$ & $\langle S\rangle$ & $\Delta\left(\operatorname{Var}_{S}\right)$ \\
\hline $101 \mathrm{st}$ & $0.0252,0.000167$ & $0.0248,1.458 \times 10^{-5}$ & $0.0251,1.454 \times 10^{-5}$ & 16.755 & 0.984 & 27.091 & 1.357 \\
\hline $102 \mathrm{nd}$ & $0.0335,0.000210$ & $0.0335,1.540 \times 10^{-5}$ & $0.0343,9.818 \times 10^{-6}$ & 17.632 & 0.856 & 37.773 & 1.403 \\
\hline $103 \mathrm{rd}$ & $0.0423,0.000194$ & $0.0425,1.544 \times 10^{-5}$ & $0.0429,9.014 \times 10^{-6}$ & 18.206 & 0.959 & 39.091 & 1.410 \\
\hline $104 \mathrm{th}$ & $0.0431,0.000171$ & $0.0430,1.262 \times 10^{-5}$ & $0.0435,7.830 \times 10^{-6}$ & 19.774 & 1.080 & 40.842 & 1.397 \\
\hline $105 \mathrm{th}$ & $0.0340,0.000108$ & $0.0339,8.927 \times 10^{-6}$ & $0.0345,6.059 \times 10^{-6}$ & 20.056 & 1.169 & 41.526 & 1.651 \\
\hline $106 \mathrm{th}$ & $0.0455,0.000128$ & $0.0455,1.298 \times 10^{-5}$ & $0.0462,5.686 \times 10^{-6}$ & 20.944 & 1.472 & 42.474 & 1.330 \\
\hline $107 \mathrm{th}$ & $0.0458,8.080 \times 10^{-5}$ & $0.0456,1.008 \times 10^{-5}$ & $0.0461,4.358 \times 10^{-6}$ & 21.867 & 1.927 & 43.368 & 1.625 \\
\hline
\end{tabular}

TABLE V: Committee extremism statistics for the 101st-107th Houses. The first column indicates the Congress. The second gives the mean and variance of the extremism of the Representatives in that Congress. The third and fourth columns give the mean and variance under the independence assumption of, respectively, all the committees and only the standing committees (without select committees). The fifth column gives the average committee size, and the sixth gives how much larger the variance of committee extremism is than would be expected under an independence assumption (as reported in column three). That is, the variance of the committees' extremism is this factor multiplied by the variance expected if Representatives were assigned to committees independently at random. The seventh and eighth columns repeat these numbers for standing committees (compare with column four).

a rank ordering of the Representatives in the 107th House and correctly classifies $92.8 \%$ of the individual Representatives' votes.

In making the connection between the voting record and committee assignment networks, we remark that we constructed the committee assignment networks representing the 101st-107th Houses from documents obtained from the website of the U. S. House of Representatives Office of the Clerk [57], which were based on the committee assignments at the end of each Congress. The roll calls, by contrast, include votes from Representatives who subsequently died or resigned and hence were not present at the end of the session. Our networks also include Representatives (such as non-voting Delegates) who do not appear in the voting record. To combine the network structures with the political spectra (as determined using the SVD analysis), it was thus necessary to reconcile the two data sets by removing a few Representatives in each of these categories (about 5-10 from each roll call and 5-10 from 


\begin{tabular}{|c|c|c|c|}
\hline Congress & Number of votes & Outcomes correctly reconstructed & \% Individual votes reconstructed \\
\hline 101 st & 879 & $867,864,848$ & $87.6 \%$ \\
\hline $102 \mathrm{nd}$ & 901 & $892,888,850$ & $87.6 \%$ \\
\hline $103 \mathrm{rd}$ & 1094 & $1075,1072,1021$ & $88.9 \%$ \\
\hline $104 \mathrm{th}$ & 1321 & $1307,1312,1225$ & $89.3 \%$ \\
\hline $105 \mathrm{th}$ & 1166 & $1157,1164,1079$ & $89.7 \%$ \\
\hline $106 \mathrm{th}$ & 1209 & $1200,1198,1121$ & $90.6 \%$ \\
\hline $107 \mathrm{th}$ & 990 & $984,975,939$ & $92.7 \%$ \\
\hline
\end{tabular}

TABLE VI: Roll call outcome reconstruction in the 101st-107th House of Representatives using two-coordinate projections of SVDs. The first column gives the Congress, and the second indicates the total number of measures in their roll call. In the third column, we show consecutively the number of outcomes correctly identified from the unmodified reconstruction, the number correctly identified throwing out known absences and abstentions, and the number correctly identified throwing out absences and abstentions and then reconstructing individual Representatives' yeas/nays and taking a majority vote. In the fourth column, we show the percentage of individual votes correctly reconstructed (which we note increases slightly from one Congress to the next during this time period).

each network). In situations where we have incorporated political spectra into our network analysis, it is always with this slightly abridged set of Representatives. The subsequent SVD computations show little change as a result of these adjustments.

\section{APPENDIX B: COMMUNITY DETECTION ALGORITHMS}

The results in the main text of this paper use single linkage clustering to determine the community structure of the network of committees, but several other methods can also be used (see, for example, [11, 17] and references therein). It is of interest to ask whether our results are robust with respect to changes in the method employed. To address this question, we have explored three other methods: two based on "betweenness" measures calculated on the full bipartite networks of Representatives and committees and a local community detection algorithm for weighted networks, generalized from the method for 
unweighted networks introduced in [3]. As we now describe, we obtain similar groupings with these different algorithms, although with some differences, suggesting that the large-scale features (but perhaps not the details) observed in our single-linkage clustering calculations are fundamental properties of the networks and not a result of our choice of methodology.

\section{Betweenness-based community detection}

Communities can be detected in many cases using "betweenness" measures that iteratively pick out and remove high-traffic edges (or other network components) that lie on a large number of paths between vertices. Repeated application of such a procedure eventually fragments a network into components, with the entire process represented by dendrograms similar to those generated by standard hierarchical clustering [17, 38, 39].

We have performed a corresponding calculation modified slightly to respect the bipartite nature of the committee assignment networks, for which betweenness can be computed by counting the number of shortest paths between pairs of committees that traverse each edge in the network. Additionally, we compute betweenness from densities of random walks between committees rather than from geodesics (see [36]), in part because the small diameter of the network often leads to many non-unique geodesics. We use this betweenness measure in two different algorithms. In one, we sequentially remove those edges (i.e., committee assignments) with highest betweenness. In the other, we sequentially remove the nodes (i.e., Representatives) lying on the largest number of paths. Applying these two methods to the full (unweighted) bipartite committee assignment graphs avoids altogether the projection onto a one-mode network and the definition of the normalized interlock used in single linkage clustering.

Comparing the different community detection schemes, we see that the dendrogram for the 107th House determined from random-walk betweenness and edge removal (see Fig. 11) shows four levels of hierarchical organization and again reveals the tight connections between the Rules Committee (and its subcommittees) and the Select Committee on Homeland Security. We also again observe the close ties between the Intelligence Committee and its subcommittees. However, other connections seemingly apparent in the single linkage clustering dendrogram are not uncovered by this betweenness-based method. Some subcommittees are not even grouped with their parent committee; for example, near the 6 o'clock and 7 o'clock 
positions, we see a weakly grouped cluster of committees that includes (consecutively) the Forests and Forest Health Subcommittee of the Resources Committee, the Select Education Subcommittee of the Education and the Workforce Committee, and the Western Hemisphere Subcommittee of the International Relations Committee.

Strahler numbers provide a way to quantify the robustness of some of these different groupings. The dendrogram representing the community structure of the 107th House determined by random-walk edge removal has a Strahler number $S=4$. Its average Strahler number of $\tilde{S} \simeq 2.7788$ quantifies the fact that many committees break off as singletons. However, the portion with the Rules Committee and Select Committee on Homeland Security has a value of 3 . This grouping therefore gives meaningful organizational information (in that it refers to an actual clique in the network), even though the tree as a whole does not show a tremendous amount of hierarchical structure.

\section{Weighted Local Community Detection}

We have also constructed dendrograms from the one-mode committee networks using a local community-detection algorithm generalized from a method for unweighted networks developed by Bagrow and Bollt [3]. The goal of this algorithm is to find a highly connected set of nodes (a "local community") near each node and to combine these individual (potentially overlapping) communities for each node into a hierarchical community structure. We again use the network of committees weighted by normalized interlocks that we considered for single linkage clustering.

To detect communities, we start with a given House's (one-mode) adjacency matrix A, whose element $A_{i j}$ gives the normalized interlock between the $i$ th and $j$ th committees. For convenience, we further normalize these elements by the maximum normalized interlock, so that $0 \leq A_{i j} \leq 1$. We use these weights as inverse distances to compute a distance matrix $\mathbf{D}$, where the element $D_{i j}$ designates the shortest distance along any path from the $i$ th node to the $j$ th node. We then define a clustering coefficient $k$ of a selected group of $n$ nodes

as the sum of all weights within that group divided by $\frac{1}{2} n(n-1)$. In our generalization of the algorithm in [3], we define the $d$-shell of node $i$ to be all nodes within distance $d$ of $i$ according to the distance matrix. We identify the local community of the $i$ th node to be the largest $d$-shell of node $i$ with $k \geq \alpha$ for some threshold $\alpha$. As $\alpha$ is increased, the definition 
of a local community becomes more stringent and smaller cliques are obtained.

Using a "membership matrix" that encodes this combined information (see Fig. 12), we manipulate the resulting collection of local communities to produce dendrograms (this procedure is described in Ref. [3] ). An unsorted membership matrix $N$ collects the ensemble of information about the local communities of each node as originally ordered in the data. Because each subcommittee is listed with its parent committee in the data ordering, this unsorted membership matrix (Fig. $12 \mathrm{a}$ ) is already nearly block partitioned. The $j$ th element of the $i$ th row has the value 1 if node $j$ is part of $i$ 's community and the value 0 if not. We compare the values in two rows $(i$ and $k$ ) and define a distance $\Delta$ between them according to the number of times they differ:

$$
\Delta(i, l)=n-\sum_{l=1}^{n} \delta\left(N_{i l}, N_{k l}\right),
$$

where $\delta$ is 1 if $N_{i l}=N_{k l}$ and 0 if not. As discussed in Ref. [3], we then compute a sorted membership matrix $\tilde{N}$ as follows: (1) compute the distance $\Delta(i, l)$ for all rows $l>i ;(2)$ swap the row $i+1$ with the row $i_{\Delta}$ that has the smallest distance to row $i$ (equivalent to interchanging vertex labels, so columns $i$ and $i_{\Delta}$ must also be swapped); (3) repeat steps 1 and 2 for row $i+1$ and continue until there are no remaining rows to consider. Wellseparated communities in $\tilde{N}$ appear as blocks along the diagonal, and their imperfections indicate possible substructure [compare panels (a) and (b) of Fig. 12].

We obtain well-structured dendrograms over a wide range of values of the local clustering threshold parameter $\alpha$ and again observe the close connections between the Rules and Homeland Security committees in the 107th House (see, for example, the 7 o'clock position in Fig. (7). These ties between the Rules and Homeland Security committees are evident even for values of $\alpha$ for which some of the other groupings in the dendrogram have disappeared, again indicating the strength of their connection.

One can depict the network's communities (and how strongly they are connected to each other) at a given level of organization using pie charts (which provide a coarse-graining of the network reminiscent of the "cartographic" visualization of networks discussed in [20]). For example, Fig. 13 shows such a pie chart for the 107th House, with each pie representing a community and the color of each wedge indicating the parent standing or select committee of the (sub)committees therein. More generally, it is desirable to study not only separate communities in networks but also the overlap between such communities and what role that 


\begin{tabular}{|c|c|c|c|}
\hline House & Mean & Standard Deviation & Local Clustering Threshold \\
\hline 101st & 8.9325 & 3.9785 & 0.1595 \\
\hline $102 \mathrm{nd}$ & 20.3558 & 5.1660 & 0.08095 \\
\hline $103 \mathrm{rd}$ & 17.0567 & 6.0884 & 0.09895 \\
\hline $104 \mathrm{th}$ & 10.8962 & 4.2537 & 0.1131 \\
\hline $105 \mathrm{th}$ & 7.4259 & 2.9425 & 0.1640 \\
\hline $106 \mathrm{th}$ & 46.5888 & 6.6118 & 0.04582 \\
\hline $107 \mathrm{th}$ & 11.7788 & 3.3746 & 0.1179 \\
\hline $108 \mathrm{th}$ & 8.8136 & 2.6212 & 0.1490 \\
\hline
\end{tabular}

TABLE VII: Community sizes in the 101st-108th Houses at the organizational level determined by the local clustering threshold value $\alpha$ that gives the highest maximum modularity. For each Congress, we list the mean number of nodes, the standard deviation, and the value of $\alpha$.

can play in the transfer of information and ideas. Such considerations have the potential to be very interesting, as committees with strong ties to multiple communities may have a substantial level of influence with otherwise disparate groups. While practically every study of community structure in networks ignores community overlap a priori, a few researchers have begun to scrutinize this feature (see, for example, [43]). The methods that have been employed in such studies are different from those discussed here, but one can compute basic statistics from the membership matrices to get some indication of community overlap. For example, the mean community size in the 107th House at the organizational level that gives the highest maximum modularity is 11.78 nodes and the standard deviation is 3.37 nodes. (The results from other Congresses are summarized in Table VII.) By comparison, the set of communities at this organizational level (see Fig. 13) has only 8 communities, giving an average community size of $113 / 8 \approx 14.1$ committees per community. The average standing committee contains $113 / 21 \approx 5.4$ subcommittees (counting the parent committee). Community sizes vary roughly with the threshold parameter $\alpha$ (which is selected to give the highest maximum modularity), with smaller values of $\alpha$ typically yielding larger communities by construction. Using the 107th House as an example, we can see from Table VII and the combination of Figs. 7, 12, and 13 that this weighted local community detection seemingly indicates a relatively small amount of overlap between the locally-defined communities. 


\section{Direct comparison of dendrograms}

In Table I, we list for each of our methods the maximum modularity obtained for a single "cut" through the dendrogram and the average modularity over all possible cuts. A cut signifies an organizational level of a dendrogram; we depict a cut graphically using a concentric circular ring of the appropriate radius that divides inter-community links (those outside the ring) from intra-community ones. See, for example, the dendrogram in Fig. 7 and the resulting community-composition pie chart in Fig. 13, For the weighted local community detection method, we used the values of the local clustering threshold $\alpha$ (denoted $\alpha_{1}, \ldots, \alpha_{8}$ for the 101st-108th Houses) giving the dendrograms with highest maximum modularity. These values are given in TableVII. Similar modularity values are obtained over a relatively broad range of $\alpha$. To see the number of organizational levels revealed by each algorithm, we list in Table VIII the maximum, mean, and standard deviation of the local Strahler numbers for the dendrograms produced for each House. Observe, for example, that the weighted local community detection method finds the largest number of organizational levels.

We compare dendrograms at the cuts (organizational levels) corresponding to their respective maximum modularities. Table IX collects these comparisons across the different clustering algorithms considered for each of the 101st-108th Houses. We compare the algorithms in pairs, with each tabulated entry indicating the fraction of committee pairs classified in the same manner by both methods (that is, both methods identify the committee pair as belonging to the same community or both methods identify the pair as belonging to separate communities). Although we list the results from specific maximum-modularity cuts in Table IX], we obtained similar values over broad ranges of cuts in the dendrograms.

To illustrate these results, we compare the similarity scores in Table IX to the dendrograms for the 107th House produced using single linkage clustering (Fig. 5), betweennessbased edge removal (Fig. 11), and our local community detection method (Fig. 7), as well as the single linkage clustering dendrogram for the 108th House (Fig. 6). The maximummodularity cuts in these dendrograms have 28, 5, 8, and 25 communities, respectively. Several observations are evident from Table IX. For example, the betweenness-based algorithms produce results that are quantitatively similar to each other but in general less similar to the other two methods. The weighted local clustering method and single linkage clustering likewise produce similar community structures. 


\begin{tabular}{|c|c|c|c|c|}
\hline Method: & SL & EB & NB & WL \\
\hline$S_{1}, \tilde{S}_{1}, S_{\sigma_{1}}$ & $4,3.289,0.758$ & $3,2.516,0.501$ & $4,2.786,0.799$ & $5,4.638,0.552$ \\
\hline$S_{2}, \tilde{S}_{2}, S_{\sigma_{2}}$ & $4,3.203,0.686$ & $4,2.620,0.669$ & $3,2.350,0.478$ & $5,4.522,0.695$ \\
\hline$S_{3}, \tilde{S}_{3}, S_{\sigma_{3}}$ & $4,3.362,0.679$ & $4,3.064,0.872$ & $3,2.582,0.495$ & $5,4.567,0.645$ \\
\hline$S_{4}, \tilde{S}_{4}, S_{\sigma_{4}}$ & $5,4.547,0.770$ & $3,2.613,0.489$ & $3,2.604,0.491$ & $5,4.368,0.588$ \\
\hline$S_{5}, \tilde{S}_{5}, S_{\sigma_{5}}$ & $5,3.880,0.924$ & $3,2.546,0.500$ & $3,2.500,0.502$ & $5,4.639,0.585$ \\
\hline$S_{6}, \tilde{S}_{6}, S_{\sigma_{6}}$ & $4,3.626,0.575$ & $4,3.393,0.491$ & $3,2.486,0.502$ & $5,4.243,0.609$ \\
\hline$S_{7}, \tilde{S}_{7}, S_{\sigma_{7}}$ & $5,4.089,0.819$ & $4,2.779,0.799$ & $3,2.575,0.497$ & $5,4.699,0.459$ \\
\hline$S_{8}, \tilde{S}_{8}, S_{\sigma_{8}}$ & $5,4.509,0.767$ & $3,2.466,0.499$ & $3,2.492,0.500$ & $5,4.254,0.666$ \\
\hline
\end{tabular}

TABLE VIII: Horton-Strahler numbers $\left(S_{i}\right)$, mean local Horton-Strahler numbers $\left(\tilde{S}_{i}\right)$, and the standard deviation $\left(\sigma_{i}\right)$ of the local Horton-Strahler numbers for the 101st-108th Houses $(i=1$ denotes the 101st House of Representatives, etc.), with dendrograms produced using single linkage clustering (SL), random-walk betweenness with sequential edge (committee assignment) removal (EB), random-walk betweenness with sequential Representative node removal (NB), and a weighted generalization (WL) of the local community detection method of [3] with the $\alpha$ values that give the highest maximum modularity.

Even when the quantitative measure of community similarity at the preferred cuts is low, many committees of interest nevertheless get grouped similarly in dendrograms produced from multiple methods, suggesting that the observed close ties between these committees are properties of the networks themselves rather than of the algorithms used. For example, the Select Committee on Homeland Security of the 107th House is grouped with the Rules Committee and its subcommittees using single linkage clustering (Fig. [5), the weighted local community determination method (Fig. 7), and the edge-betweenness based method (Fig. 11). One can also see that the Permanent Select Committee on Intelligence and its subcommittees are grouped together by all three algorithms. The recurrence of such groupings in the dendrograms, and in the visualizations of Figs. 2, 4, further supports the 


\begin{tabular}{|c|c|c|c|c|c|c|}
\hline House & WL vs SL & WL vs EB & WL vs NB & SL vs EB & SL vs NB & EB vs NB \\
\hline 101 st & 0.8056 & 0.9123 & 0.7813 & 0.7630 & 0.6077 & 0.7613 \\
\hline $102 \mathrm{nd}$ & 0.8349 & 0.4876 & 0.5837 & 0.3988 & 0.5000 & 0.8306 \\
\hline $103 \mathrm{rd}$ & 0.7896 & 0.5463 & 0.7046 & 0.4751 & 0.6301 & 0.7289 \\
\hline 104 th & 0.8794 & 0.6961 & 0.7191 & 0.6453 & 0.6855 & 0.8767 \\
\hline 105 th & 0.8927 & 0.6433 & 0.4962 & 0.6274 & 0.4886 & 0.8221 \\
\hline 106 th & 0.7907 & 0.6762 & 0.6662 & 0.6708 & 0.5860 & 0.7988 \\
\hline 107 th & 0.8685 & 0.6775 & 0.5397 & 0.6841 & 0.5653 & 0.8274 \\
\hline 108 th & 0.8893 & 0.6975 & 0.5950 & 0.6407 & 0.5196 & 0.7876 \\
\hline
\end{tabular}

TABLE IX: Comparison of House community structure as identified using different algorithms for the 101st-108th Congresses. The numbers indicate the fraction of leaf pairs identified in the same manner in pairwise comparisons of single linkage clustering (SL), edge betweenness (EB), node betweenness (NB), and the weighted local community detection method with maximum modularity (WL). Leaf pairs are identified in the same manner in two dendrograms if, at a given organizational level, both dendrograms place them in the same subtree or both place them in different subtrees. For each House, we use the organizational level identified as having the highest maximum modularity. We obtain similar comparison values over broad ranges of cuts in the dendrograms.

claim that these connections are inherent properties of the networks themselves.

[1] R. Albert and A.-L. Barabási. Statistical mechanics of complex networks. Reviews of Modern Physics, 74:47-97, 2002.

[2] A. Arenas, L. Danon, A. Diaz-Guilera, P. M. Gleiser, and R. Guimerá. Community analysis in social networks. The European Physical Journal B - Condensed Matter, 38(2):373-380, 2003.

[3] J. P. Bagrow and E. M. Bollt. A local method for detecting communities. Physical Review E, $72(046108), 2005$.

[4] J. R. Boyce and D. P. Bischak. The role of political parties in the organization of Congress. The Journal of Law, Economics, \& Organization, 18(1):1-38, 2002.

[5] T. J. Brazill and B. Grofman. Factor analysis versus multi-dimensional scaling: binary choice 
roll-call voting and the US Supreme Court. Social Networks, 24:201-229, 2002.

[6] R. S. Burt. Structural holes and good ideas. American Journal of Sociology, 110:349-399, 2004 .

[7] R. S. Burt. Brokerage and Closure: An Introduction to Social Capital. Oxford University Press, Oxford, 2005.

[8] J. Clinton, S. Jackman, and D. Rivers. The statistical analysis of roll call data. American Political Science Review, 98(2):355-370, 2004.

[9] G. W. Cox and M. D. McCubbins. Legislative Leviathan: Party Government in the House. University of California Press, Berkeley, CA, 1993.

[10] F. P. da Costa, M. Grinfeld, and J. A. D. Wattis. A hierarchical cluster system based on Horton-Strahler rules for river networks. Studies in Applied Mathematics, 109(3):163-204, 2002.

[11] L. Danon, A. Diaz-Guilera, J. Duch, and A. Arenas. Comparing community structure identification. Journal of Statistical Mechanics: Theory and Experiment, (P09008), 2005.

[12] G. F. Davis, M. Yoo, and W. E. Baker. The small world of the American corporate elite, 1982-2001. Strategic Organization, 1(3):301-326, 2003.

[13] S. N. Dorogovtsev, A. V. Goltzev, and J. F. F. Mendes. Critical phenomena in complex networks. cond-mat/0705.0010, 2007.

[14] J. H. Fowler. Connecting the Congress: A study of legislative cosponsorship networks. Political Analysis, 14(4):454-465, 2006.

[15] J. H. Fowler. Legislative cosponsorship networks in the U.S. House and Senate. Social Networks, 28(4):456-487, 2006.

[16] T. W. Gilligan and K. Krehbiel. Collective decision-making and standing committees: An informational rationale for restrictive ammendment procedures. Journal of Law, Economics, E Organization, 3:287-335, 1987.

[17] M. Girvan and M. E. J. Newman. Community structure in social and biological networks. Proceedings of the National Academy of Sciences, 99(12):7821-7826, 2002.

[18] G. H. Golub and C. F. Van Loan. Matrix Computations. The Johns-Hopkins University Press, Baltimore, Maryland, third edition, 1996.

[19] R. Guimera, B. Uzzi, J. Spiro, and L. A. N. Amaral. Team assembly mechanisms determine collaboration network structure and team performance. Science, 308(5722):697-702, 2005. 
[20] R. Guimera and L. A. N. Amaral. Functional cartography of complex metabolic networks. Nature, 433:895-900, 2005.

[21] P. R. Haunschild. Interorganizational imitation: The impact of interlocks on corporate acquisition activity. Administrative Science Quarterly, 38:564-592, 1993.

[22] R. E. Horton. Erosional development of streams and their drainage basins: hydrophysical approach to quantitative morphology. Bulletin of the Geological Society of America, 56:275$370,1945$.

[23] A. Jakulin and W. Buntine. Analyzing the US Senate in 2003: Similarities, networks, clusters and blocs. Preprint, available at http://kt.ijs.si/aleks/Politics/us_senate.pdf, 2004.

[24] S. C. Johnson. Hierarchical clustering schemes. Psychometrica, 32:241-254, 1967.

[25] T. Kamada and S. Kawai. An algorithm for drawing general undirected graphs. Information Processing Letters, 31:7-15, 1989.

[26] D. J. Kaniewski. Create a House Select Committee on Homeland Security and Terrorism. HSI Journal of Homeland Security (see http://www.homelandsecurity.org/newjournal/articles/kaniewskilegislative.htm), February 2002 .

[27] P. A. Klinkner, editor. Midterm: The Elections of 1994 in Context (Transforming American Politics). Perseus Books, Boulder, CO, 1996.

[28] G. Kossinets and D. J. Watts. Empirical analysis of an evolving social network. Science, 311(5757):88-90, 2006.

[29] K. Krehbiel. Are Congressional committees composed of preference outliers? American Political Science Review, 84:149-163, 1990.

[30] P. Mariolis. Interlocking directorates and control of corporations: The theory of bank control. Social Science Quarterly, 56:425-439, 1975.

[31] B. Mintz and M. Schwartz. The Power Structure of American Business. University of Chicago Press, Chicago, Illinois, 1985.

[32] M. S. Mizruchi. What do interlocks do? An analysis, critique, and assessment of research on interlocking directorates. Annual Review of Sociology, 22:271-298, 1996.

[33] M. E. J. Newman. The structure and function of complex networks. SIAM Review, 45(2):167256, 2003.

[34] M. E. J. Newman. Analysis of weighted networks. Physical Review E, 70:056131, 2004. 
[35] M. E. J. Newman. Fast algorithm for detecting community structure in networks. Physical Review E, 69(066133), 2004.

[36] M. E. J. Newman. A measure of betweeenness centrality based on random walks. Social Networks, 27:39-54, 2005.

[37] M. E. J. Newman. Modularity and community structure in networks. Proceedings of the National Academy of Sciences, 103(23):8577-8582, 2006.

[38] M. E. J. Newman and M. Girvan. Mixing patterns and community structure in networks. In R. Pastor-Satorras, J. Rubi, and A. Diaz-Guilera, editors, Statistical Mechanics of Complex Networks. Springer-Verlag, Berlin, Germany, 2003.

[39] M. E. J. Newman and M. Girvan. Finding and evaluating community structure in networks. Physical Review E, (026113), 2004.

[40] M. E. J. Newman, S. H. Strogatz, and D. J. Watts. Random graphs with arbitrary degree distributions and their applications. Physical Review E, 64(026118), July 2001.

[41] W. A. Niskanen. Bureaucracy and Representative Government. Aldine-Atherton, Chicago, IL, 1971.

[42] A. Pajala, A. Jakulin, and W. Buntine. Parliamentary group and individual voting behaviour in the Finnish parliament in year 2003: A group cohesion and voting similarity analysis. Preprint, available at http://www.soc.utu.fi/valtio-oppi/mopi/misc/pajala_jakulin_buntine_vers_1.pdf, 2004.

[43] G. Palla, I. Derényi, I. Farkas, and T. Vicsek. Uncovering the overlapping community structure of complex networks in nature and society. Nature, 435:814-818, 2005.

[44] K. T. Poole. Voteview. http://voteview.com, 2005.

[45] K. T. Poole and H. Rosenthal. Congress: A Political-Economic History of Roll Call Voting. Oxford University Press, Oxford, United Kingdom, 1997.

[46] K. T. Poole and H. Rosenthal. Non-parametric unfolding of binary choice data. Political Analysis, 8:211-237, 2000.

[47] M. A. Porter, P. J. Mucha, M. E. J. Newman, and C. M. Warmbrand. A network analysis of committees in the United States House of Representatives. Proceedings of the National Academy of Sciences, 102(20):7057-7062, 2005.

[48] H. Rao, G. F. Davis, and A. Ward. Embeddedness, social identity and mobility: Why firms leave the NASDAQ and join the New York Stock Exchange. Administrative Science Quarterly, 
$45: 268-292,2000$.

[49] E. Ravasz, A. L. Somera, D. A. Mongru, Z. N. Oltvai, and A.-L. Barabasi. Hierarchical organization of modularity in metabolic networks. Science, 297(5586):1551-1555, 2002.

[50] G. L. Robins and M. Alexander. Small worlds among interlocking directors: Network structure and distance in bipartite graphs. Journal of Computational and Mathematical Organization Theory, 10:69-94, 2004.

[51] M. Sales-Pardo, R. Guimerà, A. A. Moreira, and L. A. N. Amaral. Extracting the hierarchical organization of complex systems. arXiv: 0705.1679, 2007.

[52] K. A. Shepsle and B. R. Weingast, editors. Positive Theories of Congressional Institutions: A comparison of rational choice models of Congress. University of Michigan Press, Ann Arbor, Michigan, 1995.

[53] L. Sirovich. A pattern analysis of the second Renquist U.S. Supreme Court. Proceedings of the National Academy of Sciences, 100(13):7432-7437, 2003.

[54] A. N. Strahler. Hysometric (area-altitude) analysis of erosional topology. Bulletin of the Geological Society of America, 63:1117-1142, 1952.

[55] S. H. Strogatz. Exploring complex networks. Nature, 410:268-276, 2001.

[56] The Library of Congress. Thomas: Legislative information on the internet. http://thomas.loc.gov/, 2004.

[57] J. Trandahl. Office of the Clerk, U.S. House of Representatives. http://clerk.house.gov/, 2004.

[58] University of Michigan. Inter-university consortium for political and social research. http://www.icpsr.umich.edu/, 2005.

[59] M. Useem. The Inner Circle: Large corporations and the rise of business political activity in the US and UK. Oxford University Press, Oxford, 1984.

[60] D. J. Watts. The "new" science of networks. Annual Review of Sociology, 30:243-270, 2004.

[61] The Euclidean distances in these figures arise from a Kamada-Kawai force-directed network visualization algorithm [25]. While this helps make the network topology easier to see in two-dimensional projections, these distances should not be taken too seriously.

[62] The dendrograms we produce are perfectly hierarchical by construction, and it is accordingly important to provide a precise measurement of the depth of this organization. As discussed in the main text, the levels we observe have natural interpretations based on the known hierarchical organization of the House of Representatives. 
[63] This holds for the 101st-105th Houses. The leading and second eigenvectors switch roles in the 106th and 107th Houses. 


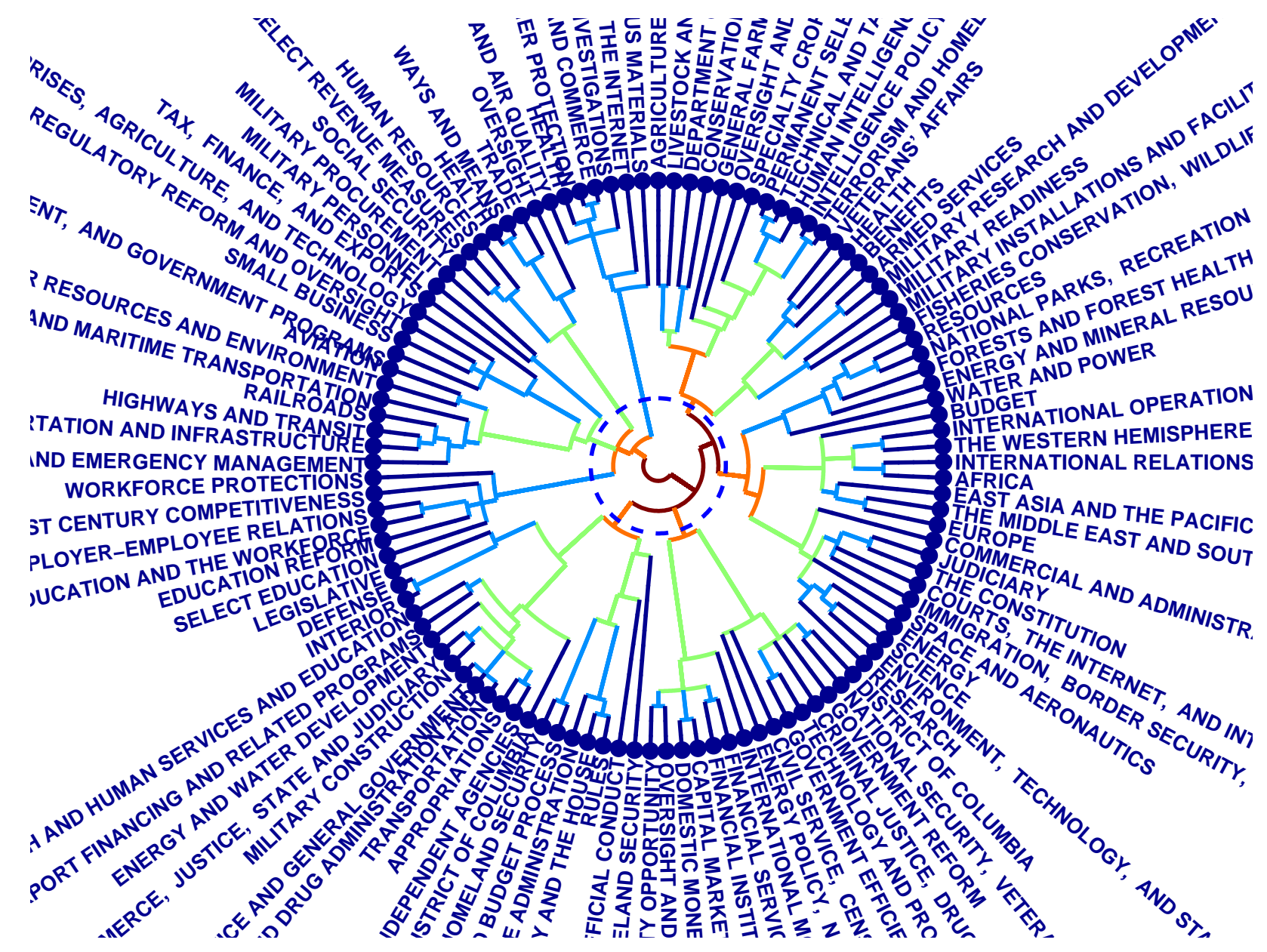

FIG. 7: (Color) Dendrogram of the committee assignment network for the 107th U.S. House of Representatives, determined using the weighted local community detection method (discussed in Appendix B) with local clustering threshold $\alpha \approx 0.1179$. This value of $\alpha$ gives the dendrogram with highest maximum modularity, as indicated by the dashed dividing ring. The dendrogram is colorcoded according to the number of hierarchical levels of each community in the tree, which is codified by Horton-Strahler numbers (discussed in the text). The Strahler numbers of the communities are calculated as one moves from the outside to the center of the tree. When two nodes of Strahler number 1 (dark blue) combine, they form a community with Strahler number 2 (light blue). We also find communities with Strahler numbers of 3 (green), 4 (orange), and 5 (maroon), indicating the five hierarchical levels in the committee assignment network of the 107th House. 

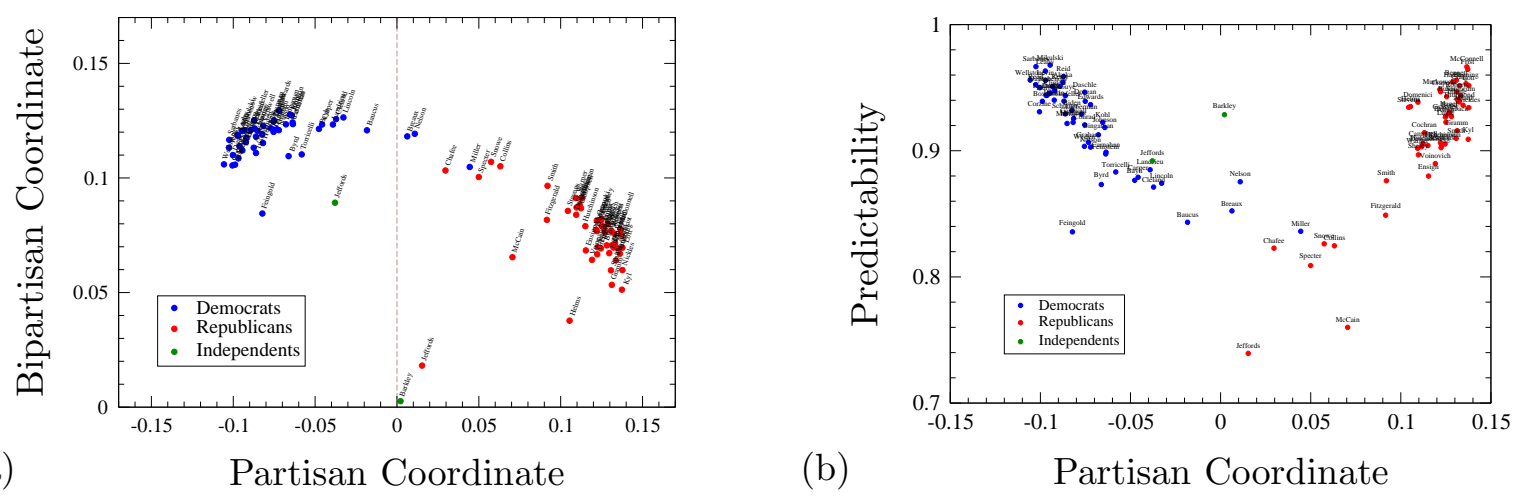

FIG. 8: (Color) Singular value decomposition (SVD) of the Senate voting record from the 107th U.S. Congress. (a) Two-dimensional projection of the voting matrix. Each point represents a projection of a single Representative's votes onto the leading two eigenvectors (labeled "partisan" and "bipartisan," as explained in the text). Democrats (blue) appear on the left and Republicans (red) are on the right. (Independents are shown in green). (b) "Predictability" of votes cast by Senators in the 107th Congress based on a two-dimensional projection of the SVD. Individual Senators range from $74 \%$ predictable to $97 \%$ predictable. 

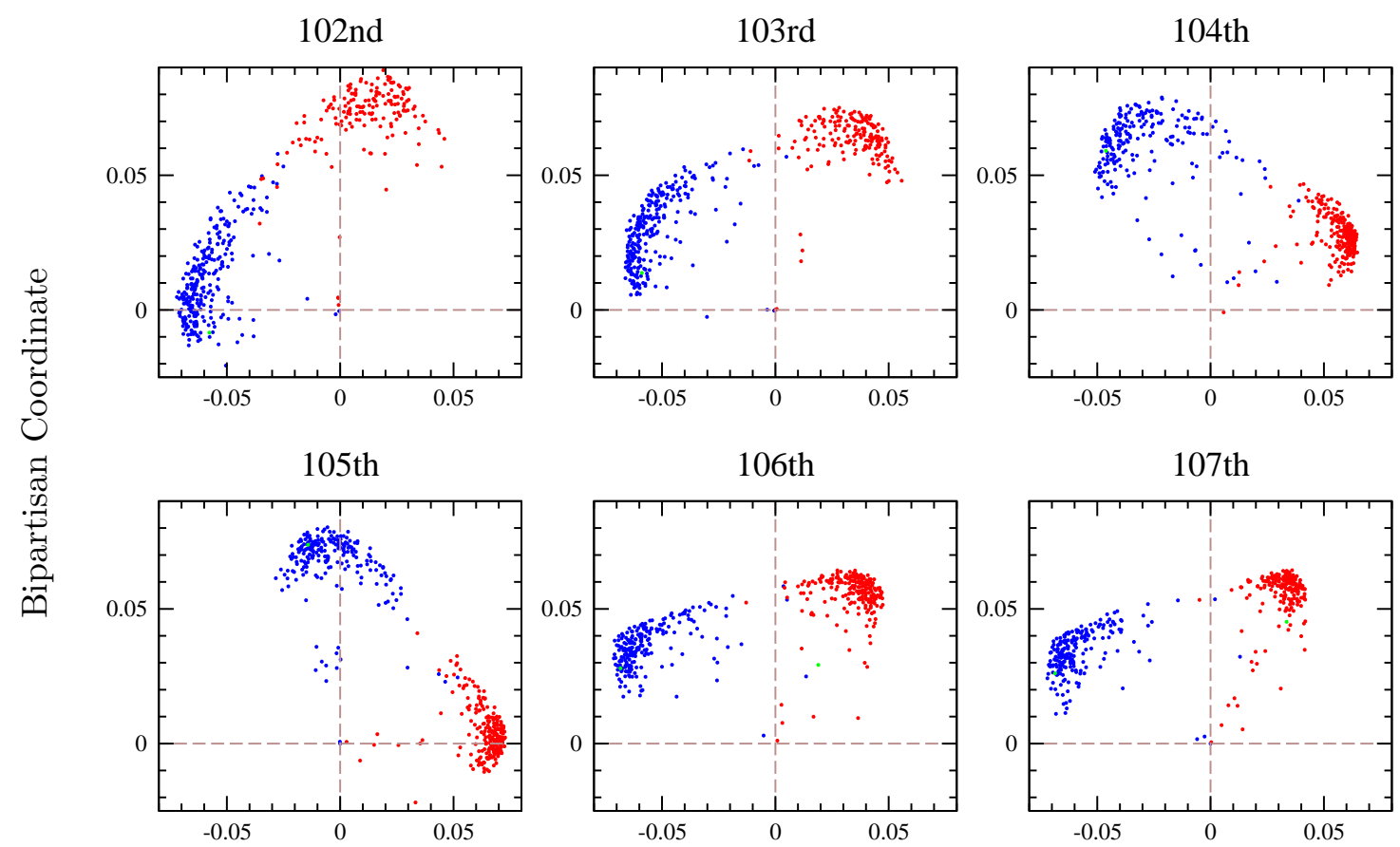

Partisan Coordinate

FIG. 9: (Color) SVD of the voting record for the House of Representatives for each of the 102nd107th U.S. Congresses. As with the Senate, Democrats (blue) appear on the left and Republicans (red) are on the right (with Independents shown in green). 


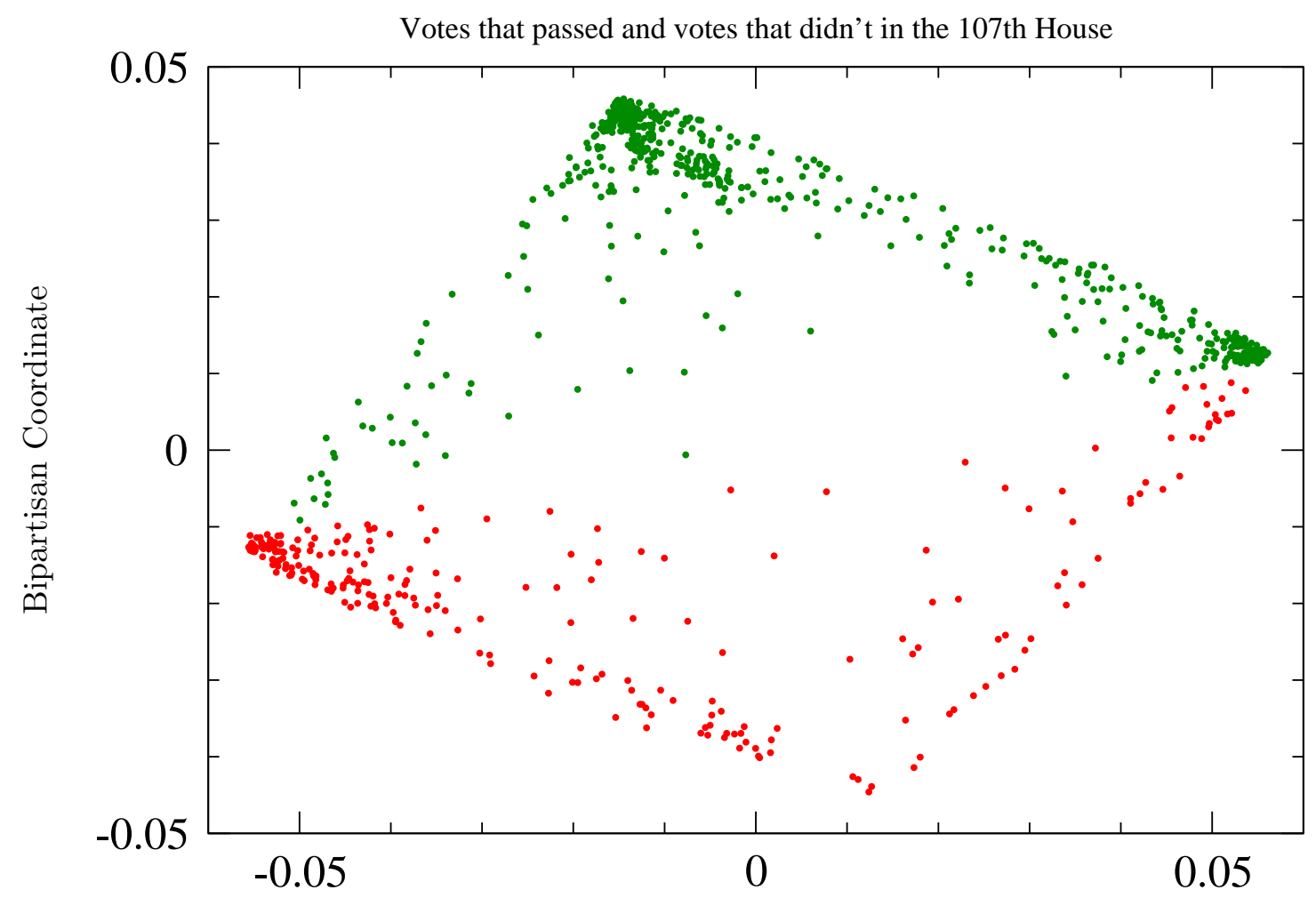

Partisan Coordinate

FIG. 10: (Color) SVD of the roll call of the 107th House projected onto the voting coordinates. Points represent projections of the votes cast on a measure onto eigenvectors associated with the leading two singular values. There is a clear separation between measures that passed (green) and those that did not (red). The four corners of the plot are interpreted as follows: measures with broad bipartisan support (north) all passed; those supported mostly by the Right (east) passed because the Republicans constituted the majority party of the 107th House; measures supported by the Left (west) failed because of the Democratic minority; and the (obviously) very few measures supported by almost nobody (south) also failed. 


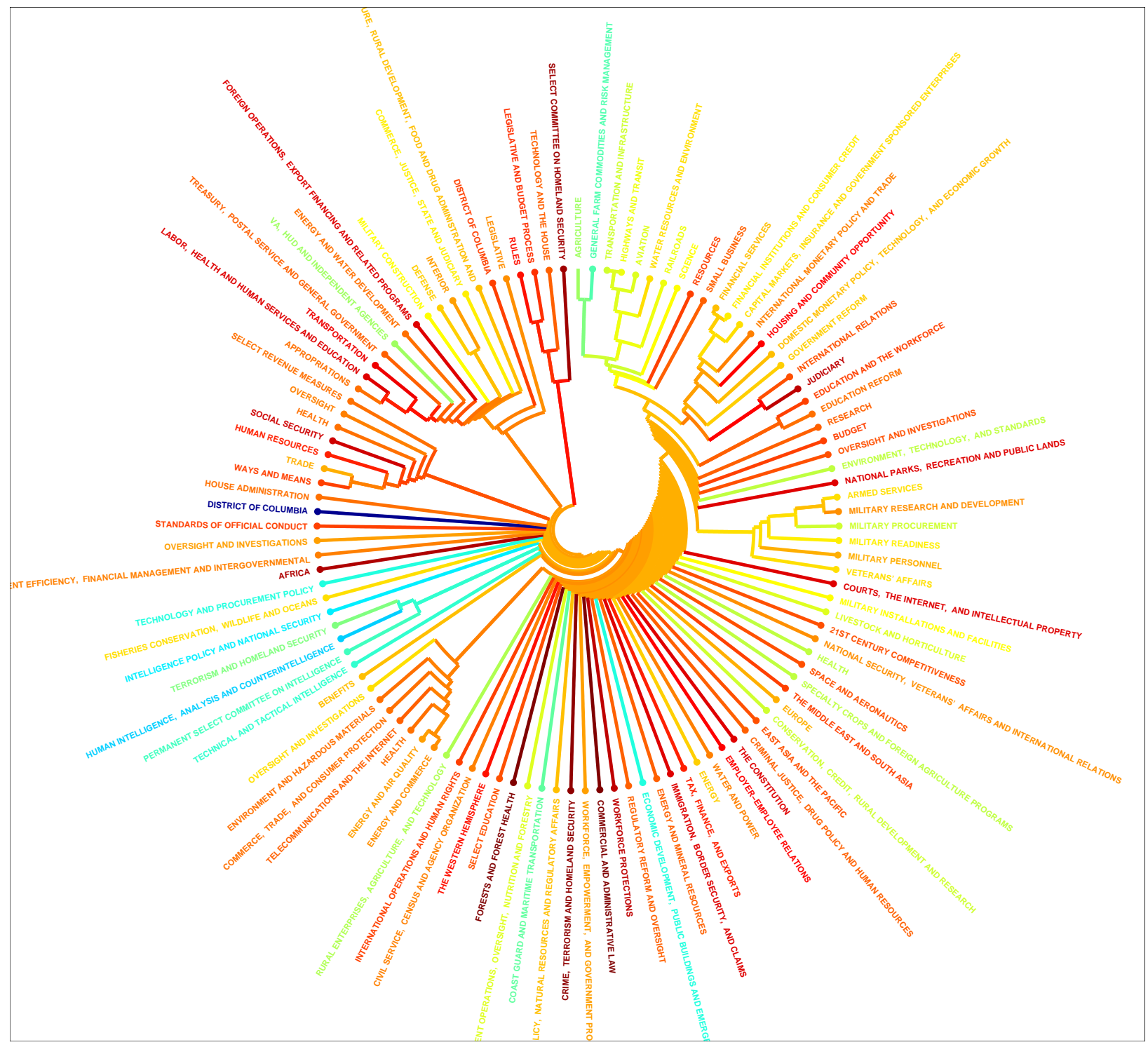

FIG. 11: (Color) Dendrogram of the committees of the 107th U.S. House of Representatives constructed by sequentially removing individual committee assignments with highest random-walk betweenness (and subsequently recomputing betweenness). Committees are listed counterclockwise around the outside of the figure in the order in which the algorithm separates them from the largest network component. Committees and groups of committees are again color-coded according to their mean extremisms. The first group of separated committees (just to the left of the 12 o'clock position) includes the Rules Committee and Select Committee on Homeland Security; this algorithm again indicates their close connection. 
(a)

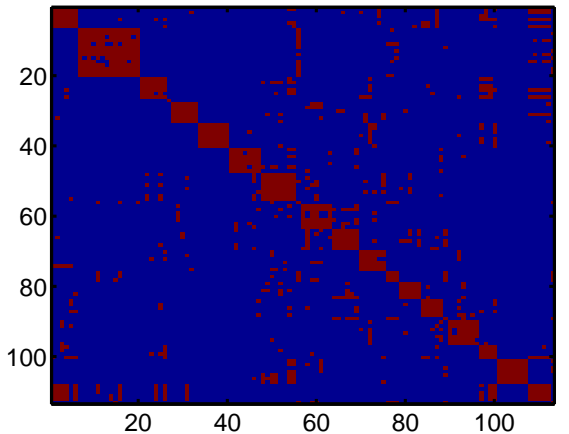

$\begin{array}{lllll}20 & 40 & 60 & 80 & 100\end{array}$ (b)

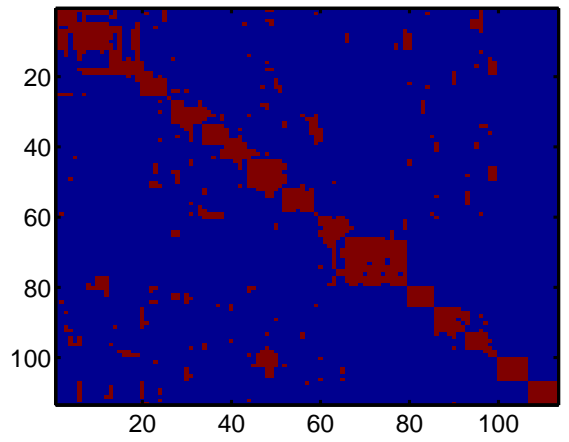

FIG. 12: (Color) Visualization of the unsorted (a) and sorted (b) membership matrices for the 107th House. The colors indicate the nearly full blocks of value 1 along the diagonal and the mostly zero-valued entries that are near the diagonal but outside of these blocks. 


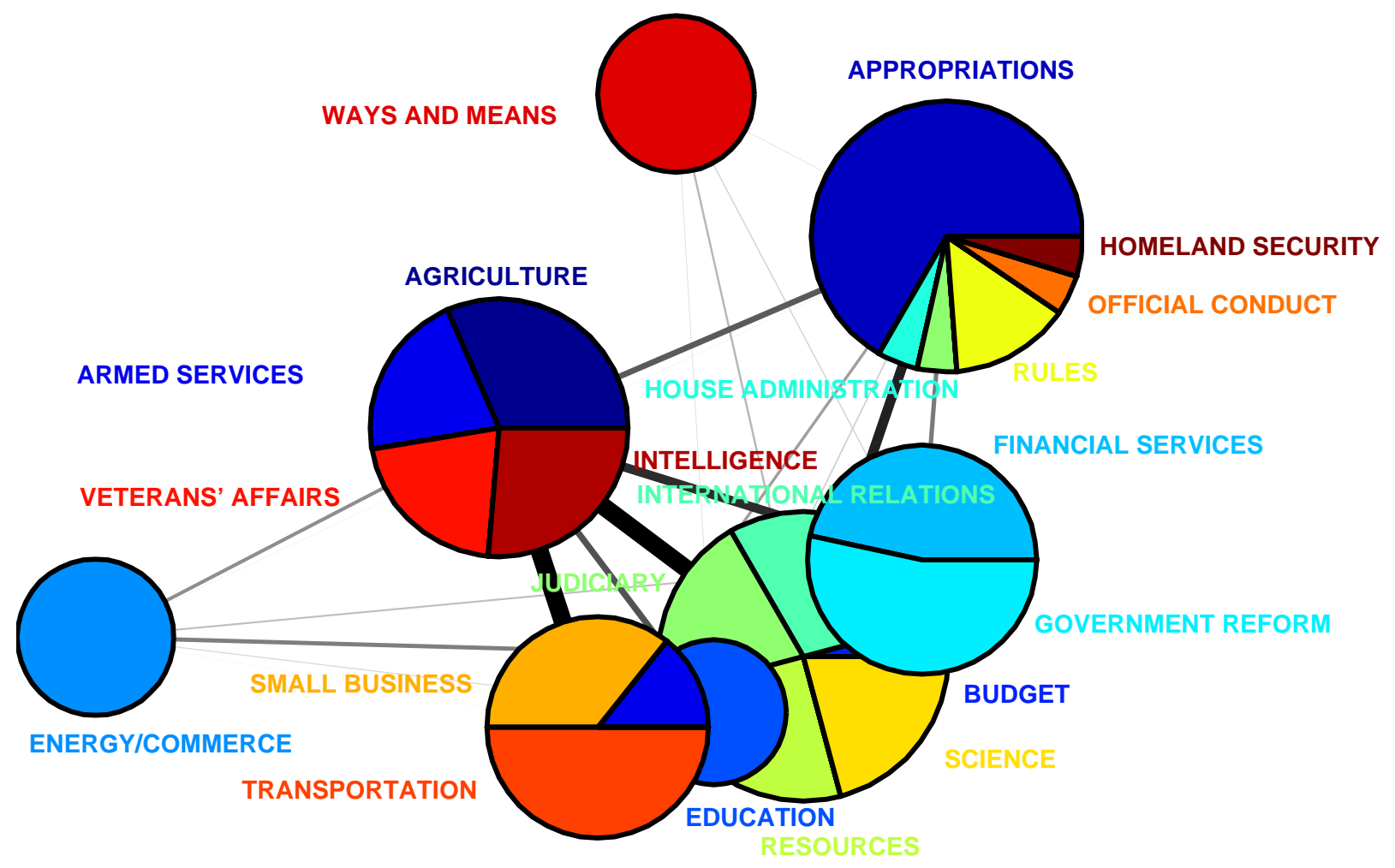

FIG. 13: (Color) Pie chart of the 107th House at the modularity-maximizing organizational level indicated by the dashed dividing ring in Fig. 7, Each pie represents a community and has wedges colored by the parent standing and select committees of the (sub)committees present in that community. The size of a wedge is determined by the number of (sub)committees it contains. Only connections between different communities are depicted (with thicker lines indicating stronger connections); the intra-community edges are not visible at this level of organization. 